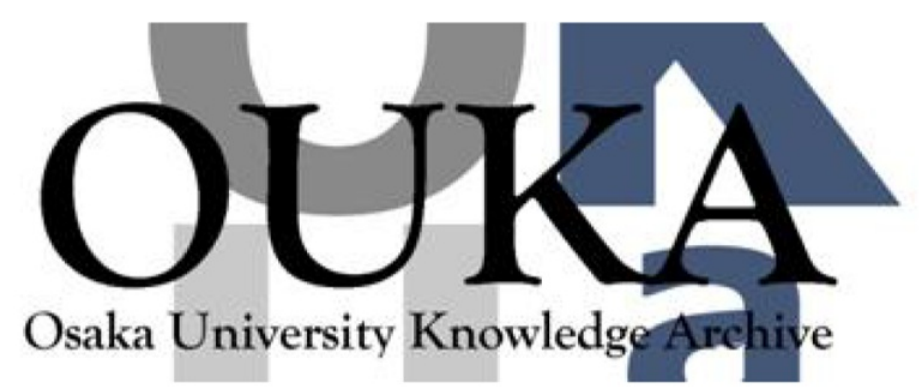

\begin{tabular}{|c|l|}
\hline Title & Information feedback in a dynamic tournament \\
\hline Author(s) & Aoyagi, Masaki \\
\hline Citation & Games and Economic Behavior \\
\hline Issue Date & 2010 \\
\hline oaire:version & AM \\
\hline URL & https://hdl. handle. net/11094/3424 \\
\hline rights & \\
\hline Note & \\
\hline
\end{tabular}

Osaka University Knowledge Archive : OUKA

https://ir. Library. osaka-u. ac. jp/

Osaka University 


\title{
Information Feedback in a Dynamic Tournament*
}

\author{
Masaki Aoyagi ${ }^{\dagger}$ \\ Osaka University \\ ISER, Osaka University, 6-1 Mihogaoka, Ibaraki, Osaka 567-0047, Japan.
}

December 4, 2009

\begin{abstract}
This paper studies the problem of information revelation in a multi-stage tournament where the agents' effort in each stage gives rise to a stochastic performance signal privately observed by the principal. The principal controls the agents' effort incentive through the use of a feedback policy, which transforms his private information into a public announcement. The optimal feedback policy is one that maximizes the agents' expected effort. The paper identifies when the principal should use the no-feedback policy that reveals no information, or the full-feedback policy that reveals all his information.

Key words: interim performance evaluation, tournament, mechanism, information revelation, Jensen's inequality.

Journal of Economic Literature Classification Numbers: C72, D82.
\end{abstract}

${ }^{*}$ This is a substantially revised version of an earlier paper under the same title (Aoyagi (2006)). I am very grateful to anonymous referees and the associate editor of this journal for helpful comments.

${ }^{\dagger}$ E-mail address: aoyagi@iser.osaka-u.ac.jp 


\section{Introduction}

As a prominent form of relative performance evaluation, tournaments have attracted considerable attention in economic theory. The main focus of the theory is on the size and allocation of rewards that maximize the performance of the competing agents, and on the comparison of the relative incentive schemes against more general forms of contracts. Beginning with the seminal work of Lazear and Rosen (1981), a partial list of the literature on this subject includes Green and Stokey (1983), Nalebuff and Stiglitz (1983), Glazer and Hassin (1988), Gradstein and Konrad (1999), Moldovanu and Sela (2001), and others. While it is common in the literature to formulate a tournament as a static game, tournaments in reality are often dynamic games in which agents make multiple effort decisions over time. ${ }^{1}$ One important consideration when designing a tournament as a dynamic game concerns the control of information during the course of play. In other words, the organizer should strategically plan how much information about agents' performance should be revealed back to them at what timing. A leading example is given by a job tournament, which typically spans multiple stages and measures workers' performance by subjective criteria such as leadership, originality, ability to work in teams, etc. Workers' performance in such a case is most appropriately described as private information of their superior or the firm's personnel department, who communicates this information back to the workers as a way of providing motivation, the process known as interim performance evaluation. Research on performance management well recognizes that inducement of the work incentive requires careful designing of information feedback. ${ }^{2}$

In this paper, we formulate a model of a dynamic tournament in which the principal receives private information about agents' performance, and then reveals as a feedback some or all of his information to the agents. The analysis is dual to that in the standard contest literature in that we fix prizes and focus exclusively on the effects of information.

In our model of a two-stage tournament, agents make private effort choices in each stage. Each agent's performance score in each stage equals the sum of his effort and a random noise term. After stage 1, the principal privately observes the stage 1 scores, and makes a public announcement about it before stage 2. A feedback policy is a mapping that transforms the stage 1 scores into the interim public announcement.

\footnotetext{
${ }^{1}$ Moldovanu and Sela (2006) provide one theoretical justification of the use of a dynamic tournament.

${ }^{2}$ See, for example, Williams (1998).
} 
An agent wins if the sum of his performance scores over the two stages exceeds that of his opponent, and is awarded a prize of a fixed value such as a promotion to a higher job rank. The optimal feedback policy is one that maximizes the principal's payoff which is an increasing function of the agents' expected efforts.

The paper presents sufficient conditions for the existence of a perfect Bayesian equilibrium (PBE) of this game, and characterizes equilibrium effort levels. The optimal feedback policy is given as follows: When the stage 2 marginal cost of effort is convex, the no-feedback policy is optimal in the class of feedback policies that admit a symmetric PBE. On the other hand, the full-feedback policy is optimal in the same class when the stage 2 marginal cost is concave. When the two agents' efforts are sufficiently complementary to each other in the principal's payoff function, the no-feedback and full-feedback policies are also optimal within the wider class of feedback policies that induce a possibly asymmetric PBE.

Existing theories provide varying intuitions on the optimal degree of information revelation. In auction theory, the so-called linkage principle by Milgrom and Weber (1982) asserts that under the affiliated distribution of signals, the seller's expected revenue is the highest when he is committed to revealing all of his private information to the bidders. ${ }^{3}$ However, the intuition furnished by the linkage principle fails in some other auction environments: Kaplan and Zamir (2000) analyze the problem of an auctioneer privately informed about bidders' valuations. In an independent private values framework, they find that the auctioneer is better off revealing the maximum of the valuations than fully revealing his information. In a model of twicerepeated common-value auctions with affiliated signals, de-Frutos and Rosenthal (1998) show that the auctioneer's expected revenue (over two auctions) is lower when information about stage 1 bids is made public than when it is not. ${ }^{4}$

The literature on dynamic models of a race also provides a closely related observation in the discussion of the closed- and open-loop formats. ${ }^{5}$ The open-loop format reveals no information to the players during a competition, whereas the closed-loop format reveals the competitors' positions publicly and instantaneously. It is often argued that the players tend to slack off in the closed-loop format since, when one player has a lead over the others, the followers cannot catch up with the leader (in

\footnotetext{
${ }^{3} \mathrm{~A}$ probability distribution is affiliated if the joint density function is log-supermodular.

${ }^{4}$ Perry and Reny (1999) report the failure of the linkage principle in a multi-object auction based on an entirely different logic.

${ }^{5}$ See, for example, Harris and Vickers (1985), and Fudenberg et al. (1983). Radner (1985) also makes a related observation in the context of a repeated principal-agent game.
} 
expected terms) by making the same level of effort as him. For example, Fudenberg et al. (1983) demonstrate the phenomenon of $\varepsilon$-preemption, where players stop making effort as soon as one of them establishes a small lead over others.

The problem of an agent's effort incentive and information is studied mainly in the context of dynamic principal-agent models. In the analysis of a repeated principal-agent game with a public performance signal, Radner (1985) considers a review strategy for the principal that evaluates the agent's performance at the end of each review phase that spans a large number of periods. He notes that inefficiency is inevitable as the agent relaxes near the end of the review phase if he realizes that his effort no longer influences the outcome of the review. ${ }^{6}$ In a paper that is closely related to the present one, Lizzeri et al. (2002) study a twostage principal-agent problem where the agent's performance information is the principal's private information as in the present paper. Comparison is made on the agent's effort and the principal's payoff when the stage 1 performance (which is either a success or a failure) is revealed to the agent and when it is not. ${ }^{7}$ They find that the revelation of information leads to a higher expected effort under the fixed wage profile and a quadratic cost function, but that the no-revelation scheme implements the same expected effort less expensively when the wage profile itself can be adjusted simultaneously. This independent finding by Lizzeri et al. (2002) complements the present analysis. ${ }^{8}$

Recently, the subjects of interim performance evaluation and information feedback are beginning to receive more attention. A partial list of papers on the subjects includes Ederer (2004), Gershkov and Perry (2006), Goltsman and Mukherjee (2006), Yildirim (2005), and Wang and Zhang (2007). ${ }^{9}$ Ederer (2004) extends the analysis of the present paper to include the possibility of private ability types. Gershkov and Perry (2006) discuss the interaction of feedback and the relative importance of the outcome of each stage tournament when the principal can only observe the identity of the leader. Goltsman and Mukherjee (2006) analyze a model with binary outcomes. Yildirim (2005) examines the agents' incentive to reveal informa-

\footnotetext{
${ }^{6}$ Also in a repeated environment, Abreu et al. (1991) analyze the problem of inducing effort when the accuracy of monitoring changes.

${ }^{7}$ With the binary private signal, these are the only (deterministic) feedback policies in Lizzeri et al. (2002).

${ }^{8}$ See Section 4 for more discussion on Lizzeri et al. (2002) as well as some other papers mentioned below.

${ }^{9}$ These papers came to my attention after the first draft of this paper was completed.
} 
tion about their interim performance. Wang and Zhang (2007) study information disclosure in elimination tournaments.

The paper is organized as follows: In the next section, we formulate a model of a dynamic tournament. Section 3 characterizes a PBE and provides sufficient conditions for its existence. Optimal feedback policies are studied in Section 4. We conclude in Section 5 with some discussion.

\section{Model of a Tournament}

Two risk neutral agents $i=1,2$ compete in two stages. In each stage, the agents' effort gives rise to stochastic performance scores. At the end of stage 2, the principal aggregates the scores from the two stages to determine the winner.

Formally, suppose that agent $i$ 's effort $a_{t}^{i}$ in stage $t$ is chosen from the set $\mathbf{R}_{+}$ of non-negative real numbers. Agent $i$ 's stage $t$ score $z_{t}^{i}$ is a random variable whose distribution depends on his effort $a_{t}^{i}$ in stage $t$. More specifically, we assume that $z_{t}^{i}=a_{t}^{i}+\varepsilon_{t}^{i}$ for a real-valued random variable $\varepsilon_{t}^{i}$. Let $\xi_{t}$ be the joint density of $\varepsilon_{t}=\left(\varepsilon_{t}^{1}, \varepsilon_{t}^{2}\right)$ over $\mathbf{R}^{2}$. We assume that $\xi_{t}$ is strictly positive, twice continuously differentiable, and symmetric in the sense that $\xi_{t}\left(\varepsilon_{t}\right)=\xi_{t}\left(\hat{\varepsilon}_{t}\right)$ when $\hat{\varepsilon}_{t}^{1}=\varepsilon_{t}^{2}$ and $\hat{\varepsilon}_{t}^{2}=\varepsilon_{t}^{1}$. Assume also that $\varepsilon_{1}$ and $\varepsilon_{2}$ are independent over stages. Let $\mu_{t}=\varepsilon_{t}^{1}-\varepsilon_{t}^{2}$ be the difference of the noise levels between the two agents in stage $t$, and denote the density of $\mu_{t}$ by $\phi_{t}$ and the corresponding cumulative distribution by $\Phi_{t}$. $\phi_{t}$ can be expressed in terms of $\xi_{t}$ as

$$
\phi_{t}\left(\mu_{t}\right)=\int_{\mathbf{R}} \xi_{t}\left(u, u-\mu_{t}\right) d u
$$

and is symmetric around zero: $\phi_{t}\left(\mu_{t}\right)=\phi_{t}\left(-\mu_{t}\right)$ for any $\mu_{t}$. Now define

$$
x_{t}=z_{t}^{1}-z_{t}^{2}=a_{t}^{1}-a_{t}^{2}+\mu_{t}
$$

to be agent 1's lead over agent 2 in terms of the stage $t$ scores. ${ }^{10}$ We assume that the principal observes $x_{t}$ after stage $t(t=1,2) .{ }^{11}$ Using $\phi_{t}$, we can write the density of $x_{t}$ under the action profile $a_{t}=\left(a_{t}^{1}, a_{t}^{2}\right)$ as

$$
\phi_{t}\left(x_{t}-a_{t}^{1}+a_{t}^{2}\right)
$$

\footnotetext{
${ }^{10}$ In this formulation, hence, $x_{t}$ changes linearly with the difference of efforts $a_{t}^{1}-a_{t}^{2}$. The conclusions of the paper continue to hold with minor modifications if $x_{t}=\lambda\left(a_{t}^{1}-a_{t}^{2}\right)+\mu_{t}$ for some differentiable function $\lambda: \mathbf{R} \rightarrow \mathbf{R}$ such that $\lambda(0)=0$ and $\lambda(d)=-\lambda(-d)$ for any $d>0$.

${ }^{11}$ See the discussion later on this assumption.
} 
Let $x=x_{1}+x_{2}$ be the aggregate lead of agent 1 over agent 2. Agent 1 wins if $x>0$, and agent 2 wins if $x<0$. Each agent wins with equal probability in the (probability zero) event of a tie $x=0$. Let $\mu=\mu_{1}+\mu_{2}$ represent the noise in $x$, and $\bar{\phi}$ denote its density:

$$
\bar{\phi}(x)=\int_{\mathbf{R}} \phi_{1}(x-u) \phi_{2}(u) d u .
$$

Each agent derives one unit of positive utility from winning the prize (e.g., promotion to a higher job rank), and incurs disutility from effort. The cost of effort in stage $t$ is described by a twice differentiable cost function $c_{t}: \mathbf{R}_{+} \rightarrow \mathbf{R}_{+}$. Accordingly, agent $i$ 's overall utility equals $1-\sum_{t=1}^{2} c_{t}\left(a_{t}^{i}\right)$ if he wins, and $-\sum_{t=1}^{2} c_{t}\left(a_{t}^{i}\right)$ otherwise. Throughout, we assume that in each stage $t=1,2$, the marginal cost of effort equals zero at no effort, and is strictly increasing:

$$
c_{t}^{\prime}(0)=0, \quad \inf _{a \in \mathbf{R}_{+}} c_{t}^{\prime \prime}(a)>0 .
$$

The principal's payoff, on the other hand, is a function of both agents' efforts over the two stages: $V\left(a_{1}^{1}, a_{1}^{2}, a_{2}^{1}, a_{2}^{2}\right)$. The function $V: \mathbf{R}_{+}^{4} \rightarrow \mathbf{R}$ is assumed to be increasing $\left(V(\hat{a}) \geq V(a)\right.$ if $\hat{a}_{t}^{i} \geq a_{t}^{i}$ for each $\left.t, i=1,2\right)$, and symmetric with respect to the agents $\left(V(\hat{a})=V(a)\right.$ if $\hat{a}_{t}^{1}=a_{t}^{2}$ and $\hat{a}_{t}^{2}=a_{t}^{1}$ for $\left.t=1,2\right)$. Furthermore, we assume that when the stage 2 efforts are symmetric $a_{2}^{1}=a_{2}^{2}=u, V\left(a_{1}, a_{2}\right)$ is an increasing affine function of $u$. In other words, there exist functions $A: \mathbf{R}_{+}^{2} \rightarrow \mathbf{R}_{+}$ and $B: \mathbf{R}_{+}^{2} \rightarrow \mathbf{R}$ such that for any $a_{1} \in \mathbf{R}_{+}^{2}$ and $u \in \mathbf{R}_{+}$,

$$
V\left(a_{1}, a_{2}\right)=A\left(a_{1}\right) u+B\left(a_{1}\right) \quad \text { if } a_{2}=(u, u) .
$$

In essence, (3) ensures that when the stage 2 effort profile is symmetric, the principal cares only about their expected values. When the payoff function is time separable, for example, then (3) holds when the stage 2 payoff is homogeneous of degree one in the agents' stage 2 efforts. Leading examples of this case include

$$
V(a)=\sum_{t=1}^{2}\left(a_{t}^{1}+a_{t}^{2}\right), \quad V(a)=\sum_{t=1}^{2} \min \left\{a_{t}^{1}, a_{t}^{2}\right\},
$$

and more generally, the CES family $V(a)=\sum_{t}\left\{\left(a_{t}^{1}\right)^{m}+\left(a_{t}^{2}\right)^{m}\right\}^{1 / m}(m \leq 1, m \neq 0)$. Since the principal's payoff may contain more information about the agents' efforts than $x$, which determines the winner of the tournament, we suppose that the payoff is observed only ex post. 
Each agent's effort $a_{t}^{i}$ is his private information and observed by neither the principal nor the other agent. On the other hand, the principal privately observes $x_{t}=z_{t}^{1}-z_{t}^{2}$ in each stage $t$ and reveals either whole or part of his private information $x_{1}$ after stage 1 . Specifically, suppose that the principal makes a public announcement $y$ about $x_{1}$ at the end of stage 1. Formally, a feedback policy (or simply a policy) is a pair of the set of possible announcements $Y$, and a measurable mapping $f: \mathbf{R} \rightarrow Y$, which chooses the announcement $y=f\left(x_{1}\right)$ as a function of signal $x_{1}$. For simplicity, reference to $Y$ will be omitted and the mapping $f$ alone will be called a feedback policy in what follows. It is understood that $Y=\left\{f\left(x_{1}\right): x_{1} \in \mathbf{R}\right\}$ so that $f$ is a surjection. The announcement $y$ is credible in the sense that the principal publicly announces his feedback policy $f$ before stage 1 and uses it to generate $y$ for any signal $x_{1} \cdot{ }^{12}$ The principal's objective is to maximize his expected payoff by controlling $f$. Although we will restrict our analysis to deterministic feedback policies, the paper's conclusions hold even when we allow for stochastic feedback policies, which choose the announcement $y$ as a function of $z_{1}$ and some (exogenous) random variable.

As mentioned above, we suppose that the principal observes only the difference $x_{1}=z_{1}^{1}-z_{1}^{2}$ between the performance scores and not the scores themselves. This is consistent with our supposition that the winner of the tournament is determined by the difference in the aggregate scores $x=x_{1}+x_{2}$. It is also justified by the observation that the difference in performance levels is often much easier to capture and evaluate than the levels themselves. This is particularly true when, for example, the two scores are highly correlated due to the presence of a large, common random shock. Technical aspects of this assumption are discussed after Theorem 3.3 in the next section, and the consequence of alternative weaker requirements on $f$ is analyzed in the Appendix. ${ }^{13}$

Given any announcement $y \in Y$, let $f^{-1}(y)=\left\{x_{1} \in \mathbf{R}: f\left(x_{1}\right)=y\right\}$ denote the inverse image of the (singleton) set $\{y\}$ under $f$. In what follows, we will restrict attention to feedback policies that satisfy the following regularity condition: A feedback policy $f$ is regular if for any $y \in Y, f^{-1}(y) \subset \mathbf{R}$ either has positive (Lebesgue) measure, or is countable. No other restriction is placed on the feedback policy. For example, each announcement $y \in Y$ may simply contain the name of the

\footnotetext{
${ }^{12}$ In other words, the principal commits to his policy. See Section 5 for more discussion.

${ }^{13}$ The assumption that feedback depends only on $x_{1}$ is required only for the analysis of optimal feedback policies. The characterization and existence of an equilibrium (Theorems 3.1 and 3.2) hold more generally. See the Appendix.
} 
leader, or it may be an interval in $\mathbf{R}$ which indicates the range of $x_{1}=z_{1}^{1}-z_{1}^{2}$.

As mentioned in the Introduction, some simple feedback policies will play an import role in our analysis. In particular, the no-feedback policy sends the same message regardless of $x_{1}$, and the full-feedback policy reveals $x_{1}$ completely. Between these two are numerous policies that reveal an intermediate amount of information. For example, the following policy reveals full information when the lead is within some range $(-b, b)(b>0)$, but nothing otherwise: $Y=(-b, b) \cup\{N\}$, and

$$
f\left(x_{1}\right)= \begin{cases}x_{1} & \text { if }\left|x_{1}\right|<b \\ N & \text { otherwise }\end{cases}
$$

Of course, the agents hearing the announcement $N$ under this policy would know that $\left|x_{1}\right| \geq b$.

Given any policy $f$, agent $i$ 's history $h^{i}$ after stage 1 is the information available to agent $i$ at the end of stage $1: h^{i}$ consists of his own effort choice $a_{1}^{i}$, and the public announcement $y$ by the principal. Agent $i$ 's (pure) strategy $\sigma^{i}$ is a pair $\left(\sigma_{1}^{i}, \sigma_{2}^{i}\right)$, where $\sigma_{1}^{i} \in \mathbf{R}_{+}$is the effort choice for stage 1 , and $\sigma_{2}^{i}: \mathbf{R}_{+} \times Y \rightarrow \mathbf{R}_{+}$is a mapping that specifies the stage 2 effort after each possible history $h^{i}=\left(a_{1}^{i}, y\right)$. Given the strategy profile $\sigma$, let $\pi_{2}^{i}\left(a_{2}^{i} \mid \sigma, h_{1}^{i}\right)$ denote agent $i$ 's expected payoff in stage 2 (payoff from the possible prize minus the cost of stage 2 effort) when he chooses $a_{2}^{i}$ in stage 2 , his history in stage 1 is $h_{1}^{i}$, and agent $j$ plays according to the strategy $\sigma^{j}$ in both stages. Likewise, let $\pi_{1}^{i}\left(a_{1}^{i} \mid \sigma\right)$ denote agent $i$ 's overall expected payoff when he chooses $a_{1}^{i}$ in stage 1 and plays according to $\sigma_{2}^{i}$ in stage 2 , and agent $j$ plays according to $\sigma^{j}$ in both stages. Throughout, we consider a perfect Bayesian equilibrium (PBE) of this tournament game in which each agent's effort choice is sequentially rational in the sense that it is optimally chosen even after an off-equilibrium effort choice in stage 1 . In the discussion of PBE, we omit reference to an agents' belief which is uniquely determined under the assumption that the distribution $\xi_{1}$ of noise $\varepsilon_{1}$ has full support. Given an equilibrium strategy $\sigma^{i}=\left(\sigma_{1}^{i}, \sigma_{2}^{i}\right)$ of agent $i$ and announcement $y \in Y$, we define

$$
\sigma_{2}^{i}(y)=\sigma_{2}^{i}\left(\sigma_{1}^{i}, y\right)
$$

to be $i$ 's stage 2 effort on the equilibrium path following announcement $y$. When $\sigma$ is given, $E\left[\cdot \mid a_{1}^{i}, y\right]$ denotes the expectation conditional on the public announcement $f\left(x_{1}\right)=y$ when agent $i$ chooses action $a_{1}^{i}$ while agent $j$ chooses $\sigma_{1}^{j}$ in stage 1 . Likewise, $E[\cdot \mid y]=E\left[\cdot \mid \sigma_{1}^{i}, y\right]$ denotes the expectation conditional on $f\left(x_{1}\right)=y$ 
when the stage 1 effort profile is $\sigma_{1}=\left(\sigma_{1}^{1}, \sigma_{1}^{2}\right)$. The unconditional expectations $E\left[\cdot \mid a_{1}^{i}\right]$ and $E[\cdot]$ are defined in a similar manner.

Let $v(\sigma, f)$ denote the principal's expected payoff in a PBE $\sigma$ under the feedback policy $f$ :

$$
v(\sigma, f)=E_{z_{1}}\left[V\left(\sigma_{1}, \sigma_{2}\left(f\left(x_{1}\right)\right)\right)\right] .
$$

The principal's objective is to maximize $v(\sigma, f)$ by choosing a feedback policy $f$ and inducing a $\mathrm{PBE} \sigma$ under $f$.

\section{Equilibrium Effort Levels}

We begin by deriving the essential marginal equation in stage 2 . When the stage 1 score is $z_{1}$ and the stage 2 effort profile is $\left(a_{2}^{1}, a_{2}^{2}\right)$, the probability that agent 1 wins is given by

$$
1-\Phi_{2}\left(-x_{1}-a_{2}^{1}+a_{2}^{2}\right)=\Phi_{2}\left(x_{1}+a_{2}^{1}-a_{2}^{2}\right),
$$

where $x_{1}=z_{1}^{1}-z_{1}^{2}$, and the equality follows from the symmetry of $\phi_{2}$ around 0 : $\Phi_{2}(x)=1-\Phi_{2}(-x)$ for any $x$. Hence, given the history $h_{1}^{1}=\left(a_{1}^{1}, y\right)$ of his own action and public announcement, agent 1's expected payoff in stage 2 can be written as:

$$
\begin{aligned}
\pi_{2}^{1}\left(a_{2}^{1} \mid \sigma, h_{1}^{1}\right) & =E_{z_{1}, \varepsilon_{2}}\left[\mathbf{1}_{\left\{z_{1}^{1}+a_{2}^{1}+\varepsilon_{2}^{1}>z_{1}^{2}+\sigma_{2}^{2}(y)+\varepsilon_{2}^{2}\right\}} \mid a_{1}^{1}, y\right]-c_{2}\left(a_{2}^{1}\right) \\
& =E_{z_{1}}\left[\Phi_{2}\left(x_{1}+a_{2}^{1}-\sigma_{2}^{2}(y)\right) \mid a_{1}^{1}, y\right]-c_{2}\left(a_{2}^{1}\right) .
\end{aligned}
$$

Taking the derivative with respect to $a_{2}^{1}$, we see that the sequentially rational choice of effort $a_{2}^{1}=\sigma_{2}^{1}\left(a_{1}^{1}, y\right)$ in stage 2 should satisfy the first-order condition

$$
E_{z_{1}}\left[\phi_{2}\left(x_{1}+\sigma_{2}^{1}\left(a_{1}^{1}, y\right)-\sigma_{2}^{2}(y)\right) \mid a_{1}^{1}, y\right]=c_{2}^{\prime}\left(\sigma_{2}^{1}\left(a_{1}^{1}, y\right)\right) .
$$

When $a_{1}^{1}$ equals the equilibrium effort choice $\sigma_{1}^{1}$ in stage 1 , hence, the effort choice $\sigma_{2}^{1}(y)=\sigma_{2}^{1}\left(\sigma_{1}^{1}, y\right)$ on the path of play in stage 2 should satisfy

$$
E_{z_{1}}\left[\phi_{2}\left(x_{1}+\sigma_{2}^{1}(y)-\sigma_{2}^{2}(y)\right) \mid y\right]=c_{2}^{\prime}\left(\sigma_{2}^{1}(y)\right) .
$$

This is the marginal equation for agent 1 in stage 2 that balances the expected marginal increment in the probability of winning with the marginal disutility of effort. The corresponding condition for agent 2 is given by

$$
E_{z_{1}}\left[\phi_{2}\left(-x_{1}-\sigma_{2}^{1}(y)+\sigma_{2}^{2}(y)\right) \mid y\right]=c_{2}^{\prime}\left(\sigma_{2}^{2}(y)\right) .
$$


With the symmetry of $\phi_{2}$, the expected marginal increments on the left-hand sides of (4) and (5) are indeed the same, and so are $\sigma_{2}^{1}(y)$ and $\sigma_{2}^{2}(y)$. With the two agents' efforts canceling each other, the expected marginal increment reduces to $E\left[\phi_{2}\left(x_{1}\right) \mid y\right]$ in equilibrium. The following theorem summarizes this argument and also describes the first-order conditions for the stage 1 effort in any pure PBE. Recall that $\bar{\phi}$ denotes the density of the aggregate noise $\mu_{1}+\mu_{2}=\varepsilon_{1}^{1}-\varepsilon_{1}^{2}+\varepsilon_{2}^{1}-\varepsilon_{2}^{2}$.

Theorem 3.1. Suppose that

$$
\sup _{x \in \mathbf{R}} \phi_{2}^{\prime}(x)<\inf _{a \in \mathbf{R}_{+}} c_{2}^{\prime \prime}(a) .
$$

If $\sigma$ is a pure PBE under any feedback policy $f$, then for any $y \in Y$,

$$
\sigma_{2}^{1}(y)=\sigma_{2}^{2}(y)=a_{2}^{*}\left(\sigma_{1}, y\right) \equiv\left(c_{2}^{\prime}\right)^{-1}\left(E_{z_{1}}\left[\phi_{2}\left(x_{1}\right) \mid f\left(x_{1}\right)=y\right]\right) .
$$

If, in addition, $\sigma_{1}^{1}, \sigma_{1}^{2}>0$, then

$$
\begin{aligned}
& c_{1}^{\prime}\left(\sigma_{1}^{1}\right)=\bar{\phi}\left(\sigma_{1}^{1}-\sigma_{1}^{2}\right)+\int_{\mathbf{R}} c_{2}\left(a_{2}^{*}\left(\sigma_{1}, f\left(x_{1}\right)\right)\right) \phi_{1}^{\prime}\left(x_{1}-\sigma_{1}^{1}+\sigma_{1}^{2}\right) d x_{1}, \\
& c_{1}^{\prime}\left(\sigma_{1}^{2}\right)=\bar{\phi}\left(\sigma_{1}^{1}-\sigma_{1}^{2}\right)-\int_{\mathbf{R}} c_{2}\left(a_{2}^{*}\left(\sigma_{1}, f\left(x_{1}\right)\right)\right) \phi_{1}^{\prime}\left(x_{1}-\sigma_{1}^{1}+\sigma_{1}^{2}\right) d x_{1} .
\end{aligned}
$$

Proof. See the Appendix.

Note from Theorem 3.1 that information feedback has two separate effects on the agents' incentives. First, the revealed information influences the agents' incentives by changing their beliefs. This, which we call the ex post effect of information feedback, is reflected in the conditioning event in the stage 2 effort in (6). Next, agents choose their stage 1 effort so as to influence the content of the revealed information. This is reflected in the second term on the left-hand sides of (7). We call this the strategic effect of information feedback.

As seen above, the stage 2 effort is determined through the standard marginal consideration, and the symmetry of the agents' stage 2 effort profile holds for any announcement whether the equilibrium itself is symmetric or not. It can also be seen that the expected marginal cost in stage 2 is independent of the feedback policy since by the law of iterated expectation

$$
\begin{aligned}
E_{z_{1}}\left[c_{2}^{\prime}\left(a_{2}^{*}\left(\sigma_{1}, f\left(x_{1}\right)\right)\right)\right] & =E_{z_{1}}\left[E_{\hat{z}_{1}}\left[\phi_{2}\left(\hat{x}_{1}\right) \mid f\left(\hat{x}_{1}\right)=f\left(x_{1}\right)\right]\right] \\
& =E_{z_{1}}\left[\phi_{2}\left(x_{1}\right)\right] \\
& =\bar{\phi}(0) .
\end{aligned}
$$


One implication of (6) is as follows. Suppose for simplicity that $f$ is the fullfeedback policy: $f\left(x_{1}\right)=x_{1}$. In this case, $\sigma_{2}^{i}\left(z_{1}\right)=\left(c_{2}^{\prime}\right)^{-1}\left(\phi_{2}\left(x_{1}\right)\right)$ as is readily verified. It follows that the stage 2 effort is maximized when $\phi_{2}\left(x_{1}\right)$ is the largest. If $\phi_{2}$ is unimodal at the origin as in the case of the normal distribution, hence, the stage 2 effort is a monotone decreasing function of $\left|x_{1}\right|$. Such a specification supports the common intuition that the closer the competition, the higher the efforts the agents exert. Note, however, that this intuition fails when, for example, $\phi_{2}$ is bimodal so that $\phi_{2}(x)=\phi_{2}(-x)>\phi_{2}(0)$ for some $x>0$.

We next turn to the existence of a pure PBE. As discussed in Nalebuff and Stiglitz (1983), the existence of an equilibrium in a moral hazard tournament requires noise to be sufficiently large. Intuitively, if the noise is too small, then any infinitesimal increase in effort results in almost sure winning, making it impossible for the marginal equation to hold. The assumption below specifies how large the noise should be for the existence of an equilibrium. ${ }^{14}$

Assumption 1. Let $\kappa=\min \left\{1, \inf _{a \in \mathbf{R}_{+}} c_{1}^{\prime \prime}(a), \inf _{a \in \mathbf{R}_{+}} c_{2}^{\prime \prime}(a), \lim _{a \rightarrow \infty} c_{2}^{\prime}(a)\right\}>0$. Then

$$
\sup _{x \in \mathbf{R}} \phi_{2}(x), \sup _{x \in \mathbf{R}} \phi_{2}^{\prime}(x), \int_{\mathbf{R}^{2}}\left|\frac{\partial^{2} \xi_{1}}{\partial\left(\varepsilon_{1}^{i}\right)^{2}}(\varepsilon)\right| d \varepsilon, \int_{\mathbf{R}^{2}}\left(\frac{\partial \xi_{1}}{\partial \varepsilon_{1}^{i}}(\varepsilon)\right)^{2} \frac{1}{\xi_{1}(\varepsilon)} d \varepsilon<\frac{\kappa}{2} .
$$

Theorem 3.2. Suppose that Assumption 1 holds. Given any feedback policy $f$, there exists a pure PBE under $f$ if $(7)$ has a solution $\sigma_{1}=\left(\sigma_{1}^{1}, \sigma_{1}^{2}\right) \geq 0$.

Proof. See the Appendix.

For any set $A \subset \mathbf{R}$, define $-A=\{-x: x \in A\}$. We say that a feedback policy $f$ is symmetric if $f^{-1}\left(f\left(x_{1}\right)\right)=-f^{-1}\left(f\left(-x_{1}\right)\right)$ for any $x_{1} \in \mathbf{R}$. Note that this comprises two possibilities: When $f\left(x_{1}\right)=f\left(-x_{1}\right)$, this implies that the set

${ }^{14}$ If, for example, $\varepsilon_{t}=\left(\varepsilon_{t}^{1}, \varepsilon_{t}^{2}\right)$ has the normal distribution $N(0, \Sigma)$ where $\Sigma=\sigma^{2}\left(\begin{array}{ll}1 & \rho \\ \rho & 1\end{array}\right)$ $(t=1,2)$ so that $\phi_{t}\left(\varepsilon_{t}\right)=\frac{1}{2 \pi} \Sigma^{-1 / 2} \exp \left\{-\frac{1}{2} \varepsilon_{t}^{\prime} \Sigma \varepsilon_{t}\right\}$, then Assumption 1 holds if the variance $\sigma^{2}$ is sufficiently large. In particular, note that

$$
\int_{\mathbf{R}^{2}}\left|\frac{\partial^{2} \xi_{1}}{\partial\left(\varepsilon_{1}^{i}\right)^{2}}(\varepsilon)\right| d \varepsilon=\int_{\mathbf{R}^{2}}\left|-\frac{1}{\sigma^{2}\left(1-\rho^{2}\right)} \xi_{1}(\varepsilon)+\frac{\left(\varepsilon^{1}-\rho \varepsilon^{2}\right)^{2}}{\sigma^{4}\left(1-\rho^{2}\right)^{2}} \xi_{1}(\varepsilon)\right| d \varepsilon \leq \frac{2}{\sigma^{2}\left(1-\rho^{2}\right)},
$$

and

$$
\int_{\mathbf{R}^{2}}\left(\frac{\partial \xi_{1}}{\partial \varepsilon_{1}^{i}}(\varepsilon)\right)^{2} \xi_{1}(\varepsilon)^{-1} d \varepsilon=\int_{\mathbf{R}^{2}} \frac{\left(\varepsilon^{1}-\rho \varepsilon^{2}\right)^{2}}{\sigma^{2}\left(1-\rho^{2}\right)} \xi_{1}(\varepsilon) d \varepsilon=\frac{1}{\sigma^{2}\left(1-\rho^{2}\right)} .
$$


$f^{-1}\left(f\left(x_{1}\right)\right)$ itself is symmetric around 0 , suggesting that the inference drawn from the announcement $f\left(x_{1}\right)$ and that drawn from $f\left(-x_{1}\right)$ are the same. When $f\left(x_{1}\right) \neq$ $f\left(-x_{1}\right)$, symmetry implies that the sets $f^{-1}\left(f\left(x_{1}\right)\right)$ and $f^{-1}\left(f\left(-x_{1}\right)\right)$ are symmetric with respect to 0 , suggesting that the inference drawn from the announcement $f\left(x_{1}\right)$ is the exact opposite of that drawn from $f\left(-x_{1}\right)$. The full- and no-feedback policies are both symmetric. The focus on symmetric policies is in line with the symmetry between the two agents.

A strategy profile $\sigma$ is symmetric if the two agents choose the same effort level on the path: $\sigma_{1}^{1}=\sigma_{1}^{2}$ and $\sigma_{2}^{1}(y)=\sigma_{2}^{2}(y)$ for any $y \in Y$. We now show that every symmetric policy admits a symmetric PBE under Assumption 1. Summing the two equations of (7), we see that the stage 1 effort in a symmetric PBE (if any) must satisfy

$$
\sigma_{1}^{1}=\sigma_{1}^{2}=a_{1}^{*} \equiv\left(c_{1}^{\prime}\right)^{-1}(\bar{\phi}(0))
$$

The following theorem confirms that this $a_{1}^{*}$ is indeed the equilibrium stage 1 effort when $f$ is symmetric.

Theorem 3.3. Suppose that Assumption 1 holds. If $f$ is symmetric, there exists a unique symmetric pure PBE $\sigma$. Furthermore, for $a_{2}^{*}$ defined in (6) and $a_{1}^{*}$ defined in (10), $\sigma$ satisfies

$$
\sigma_{1}^{1}=\sigma_{1}^{2}=a_{1}^{*}
$$

and

$$
\sigma_{2}^{1}(y)=\sigma_{2}^{2}(y)=a_{2}^{*}\left(\sigma_{1}, y\right) \quad \text { for any } y \in Y \text {. }
$$

Proof. See the Appendix.

The following comments are in order on Theorem 3.3. First, the noise level required for the existence of a PBE is independent of the choice of a feedback policy. Second, by (8) and (11), in the symmetric PBE, the marginal cost of effort in stage 1 and the expected marginal cost in stage 2 both equal $\bar{\phi}(0)$. This is a very intuitive intertemporal relationship and its counterpart forms the basis of, for example, the theory of precautionary savings. Third, the stage 1 effort in symmetric PBE is independent of the choice of a feedback policy. The second and third properties above are closely related to each other, and both may fail when the PBE is asymmetric 
or when the principal observes more than just $x_{1}$. The following is a discussion on this point in some detail.

As seen from (7), the strategic effect of information feedback on agent 1's stage 1 incentive is exactly opposed with that on agent 2's incentive. In other words, whatever information may be expected regarding the difference between the stage 1 scores, if it raises agent 1's stage 1 effort incentive, it reduces agent 2's incentive, and vice versa. If the two agents' stage 1 effort choices are the same as in symmetric PBE, it must then be the case that the strategic effect is null for both agents. This explains why the stage 1 effort in symmetric PBE is the same under any feedback policy. The same observation does not hold for information regarding the aggregate performance score $s_{1}=z_{1}^{1}+z_{1}^{2}$ in stage 1 . Specifically, if the principal observes and releases information about $s_{1}$ in addition to that on $x_{1}$, any information expected on $s_{1}$ works in the same direction for the two agents. For this reason, when $f$ is a function also of $s_{1}$, the marginal cost of the agents' stage 1 effort does not necessarily equal the marginal cost of their stage 2 effort even in symmetric PBE. In the Appendix, we identify the conditions under which the strategic effect is nullified even with feedback of information on the aggregate score. Under these conditions, the conclusions in the next section on the optimal policies in symmetric PBE generalize. ${ }^{15}$

\section{Optimal Feedback Policy}

The following facts about the no-feedback and full-feedback policies are readily implied by Theorem 3.3 .

Proposition 4.1. If $\sigma$ is the (unique) symmetric pure PBE under the no-feedback policy, then the stage 1 effort equals $\sigma_{1}^{i}=a_{1}^{*}$ and the stage 2 effort equals

$$
\sigma_{2}^{i}=a_{2}^{N} \equiv\left(c_{2}^{\prime}\right)^{-1}(\bar{\phi}(0)) .
$$

Likewise, if $\sigma$ is the (unique) symmetric pure PBE under the full-feedback policy, then the stage 1 effort equals $\sigma_{1}^{i}=a_{1}^{*}$ and the expected stage 2 effort equals

$$
E\left[\sigma_{2}^{i}(y)\right]=a_{2}^{F} \equiv \int_{\mathbf{R}^{2}}\left(c_{2}^{\prime}\right)^{-1}\left(\phi_{2}\left(x_{1}\right)\right) \phi_{1}\left(x_{1}\right) d z_{1} .
$$

\footnotetext{
${ }^{15}$ On the other hand, the conclusions on asymmetric PBE do not generalize given that the discussion there is based on the fact that the two strategic effect terms in (7) cancel out each other.
} 
When $\left(c_{2}^{\prime}\right)^{-1}$ is concave or convex, Proposition 4.1 can be used to rank the no-feedback and full-feedback policies in terms of the expected stage 2 effort they induce in the symmetric PBE. Suppose for example that $\left(c_{2}^{\prime}\right)^{-1}$ is concave. Then Jensen's inequality implies the no-feedback policy induces a higher expected effort than the full-feedback policy:

$$
a_{2}^{F}=\int_{\mathbf{R}^{2}}\left(c_{2}^{\prime}\right)^{-1}\left(\phi_{2}\left(x_{1}\right)\right) \phi_{1}\left(x_{1}\right) d z_{1} \leq\left(c_{2}^{\prime}\right)^{-1}\left(\int_{\mathbf{R}^{2}} \phi_{2}\left(x_{1}\right) \phi_{1}\left(x_{1}\right) d z_{1}\right)=a_{2}^{N} .
$$

The reverse inequality holds when $\left(c_{2}^{\prime}\right)^{-1}$ is convex. This section considers generalizations of these inequalities. Given that the stage 2 efforts in any PBE are always symmetric between the two agents by (6), it follows from our assumption (3) on the principal's payoff function $V$ that his expected payoff is an increasing function of their expected stage 2 effort. ${ }^{16}$ Recall that $v(\sigma, f)$ denotes the principal's expected payoff in a PBE $\sigma$ under the feedback policy $f$.

\subsection{Symmetric Equilibrium}

Even when $f$ admits multiple symmetric pure PBE's, they all induce the same onthe-path effort by Theorem 3.1 and equation (10). In this sense, the principal's payoff is independent of the choice of a symmetric $\operatorname{PBE} \sigma$. Hence, we define

$$
\bar{v}^{*}(f)= \begin{cases}v(\sigma, f) & \text { if } f \text { admits a symmetric pure } \operatorname{PBE} \sigma \\ 0 & \text { otherwise. }\end{cases}
$$

Theorem 4.2. Suppose that Assumption 1 holds.

1. If the stage 2 marginal cost $c_{2}^{\prime}$ is convex over $\left[0,\left(c_{2}^{\prime}\right)^{-1}\left(\sup _{x \in \mathbf{R}} \phi_{2}(x)\right)\right]$, then the no-feedback policy maximizes $\bar{v}^{*}$ among all policies.

2. If the stage 2 marginal cost $c_{2}^{\prime}$ is concave over $\left[0,\left(c_{2}^{\prime}\right)^{-1}\left(\sup _{x \in \mathbf{R}} \phi_{2}(x)\right)\right]$, then the full-feedback policy maximizes $\bar{v}^{*}$ among all policies.

Proof. We only prove the case of the convex marginal cost since the proof of the other case is similar. Take any policy $f$ with a symmetric pure PBE $\sigma$. Since the stage 1 effort is the same in any symmetric PBE, it suffices to show that the expected stage 2 effort is maximized under the no-feedback policy. Since $\left(c_{2}^{\prime}\right)^{-1}$ is concave

\footnotetext{
${ }^{16}$ In the consideration of a symmetric PBE in Section 4.1, we only need the linearity of $V\left(a_{1}, a_{2}^{1}=\right.$ $\left.a_{2}^{2}=u\right)$ in $u$ for $a_{1}$ such that $a_{1}^{1}=a_{1}^{2}$.
} 
over $\left[0, \sup _{x \in \mathbf{R}} \phi_{2}(x)\right]$, it follows from Jensen's inequality and the law of iterated expectation that the expected stage 2 effort under $f$ satisfies

$$
\begin{aligned}
E_{y}\left[a_{2}^{*}\left(\sigma_{1}, y\right)\right] & =E_{y}\left[\left(c_{2}^{\prime}\right)^{-1}\left(E_{z_{1}}\left[\phi_{2}\left(x_{1}\right) \mid y\right]\right)\right] \\
& \leq\left(c_{2}^{\prime}\right)^{-1}\left(E_{y}\left[E_{z_{1}}\left[\phi_{2}\left(x_{1}\right) \mid y\right]\right]\right) \\
& =\left(c_{2}^{\prime}\right)^{-1}\left(E_{z_{1}}\left[\phi_{2}\left(x_{1}\right)\right]\right) \\
& =\left(c_{2}^{\prime}\right)^{-1}(\bar{\phi}(0)) \\
& =a_{2}^{N},
\end{aligned}
$$

where the third equality follows from the symmetry of the stage 1 effort profile $\sigma_{1}$.

The proof of Theorem 4.2 also indicates that when $c_{2}^{\prime}$ is concave (resp. convex), the no-feedback (resp. full-feedback) policy yields the lowest expected payoff to the principal. On the other hand, when the marginal cost function $c_{2}^{\prime}$ for stage 2 is linear (and hence both concave and convex), the induced effort in either stage is not affected by the feedback policy. The following corollary summarizes this immediate consequence of Theorem 3.1.

Corollary 4.3. Suppose that the stage 2 cost function is quadratic: $c_{2}(a)=\frac{1}{2} k a^{2}$ for some $k>0$. Suppose also that $\sup _{x \in \mathbf{R}} \phi_{2}^{\prime}(x)<k$. In any symmetric pure PBE $\sigma$ under $f$, the stage 1 effort equals $\sigma_{1}^{i}=a_{1}^{*}$, and the expected stage 2 effort equals $E\left[\sigma_{2}^{i}(y)\right]=\frac{1}{k} \bar{\phi}(0)$. It follows that the principal's expected payoff $v(\sigma, f)$ is independent of $f$.

Lizzeri et al. (2002) compare the no-feedback and full-feedback policies under the assumption that effort cost is a quadratic function, and state (in Lemma 1) that the expected stage 2 effort is the same with or without feedback provided that the stage 1 effort is fixed at some level. Corollary 4.3 parallels this result since in our model, the stage 1 effort is the same in symmetric PBE under any feedback policy. Lizzeri et al. (2002) then conclude that full feedback is optimal (Proposition 2 ) based primarily on the comparison of stage 1 effort under the two policies. In our terminology, this can be expressed as the higher strategic effect induced by full feedback in their model. Note that our point of comparison is reversed: The neutrality result above is based on the comparison of the expected ex post effects on stage 2 effort since the strategic effect is zero in symmetric PBE. Ederer (2004) and Goltsman and Mukherjee (2006) also reach some different conclusions on optimal 
feedback with the quadratic cost function. In a model where an agent's performance is a function not only of his effort but also of his (unknown) ability, Ederer (2004) finds that when the ability and effort work additively, full feedback and no feedback induce the same expected effort, but that when they work multiplicatively, full feedback is better than no feedback. The latter conclusion can be attributed to the agents' learning about their abilities, which Ederer (2004) refers to as motivation. In a model where each agent's effort leads to either success or failure in each stage, Goltsman and Mukherjee (2006) find that the optimal symmetric feedback policy is to reveal whether both agents have failed or at least one agent has succeeded. This non-neutrality result is likely to stem from the way performance is defined in their model.

\subsection{Asymmetric Equilibrium}

We now allow a PBE $\sigma$ to be asymmetric, and define

$$
\bar{v}(f)=\sup \{v(\sigma, f): \sigma \text { is a pure } \mathrm{PBE} \text { under } f \text { and satisfies }(7)\},
$$

with $\bar{v}(f)=-\infty$ if the corresponding strategy profile does not exist. We need some additional assumptions in order to evaluate the principal's expected payoff when the stage 1 effort profile is asymmetric. Specifically, we will identify the situations where the principal obtains a higher payoff in a symmetric PBE than in an asymmetric PBE. In such situations, the optimality of the no-feedback or full-feedback policies is obtained just as before. Intuitively, a symmetric PBE is more desirable for the principal than an asymmetric PBE if the two agents' efforts enter his payoff function in a complementary manner. In other words, we would want the principal's payoff to be higher when both agents make moderate efforts than when one agent makes a high effort and the other makes a low effort. The assumption below specifies just how much complementarity is sufficient for our conclusion.

Assumption 2. The principal's payoff function $V$ is differentiable, and for any $a=\left(\left(a_{1}^{1}, a_{1}^{2}\right),\left(a_{2}^{1}, a_{2}^{2}\right)\right) \in \mathbf{R}_{+}^{4}$ such that $a_{1}^{1}<a_{1}^{2}$ and $a_{2}^{1}=a_{2}^{2}$, we have

$$
\frac{c_{1}^{\prime \prime}\left(a_{1}^{1}\right)-2 \bar{\phi}^{\prime}\left(a_{1}^{1}-a_{1}^{2}\right)}{c_{1}^{\prime \prime}\left(a_{1}^{2}\right)+2 \bar{\phi}^{\prime}\left(a_{1}^{1}-a_{1}^{2}\right)}<\frac{\frac{\partial V}{\partial a_{1}^{1}}(a)}{\frac{\partial V}{\partial a_{1}^{2}}(a)} .
$$

It can be seen that the left-hand side of (12) represents the slope of the curve

$$
h\left(a_{1}^{1}, a_{1}^{2}\right) \equiv c_{1}^{\prime}\left(a_{1}^{1}\right)+c_{1}^{\prime}\left(a_{1}^{2}\right)-2 \bar{\phi}\left(a_{1}^{1}-a_{1}^{2}\right)=0
$$


in the $\left(a_{1}^{1}, a_{1}^{2}\right)$-plane, which equals the sum of the two first-order conditions in (7). On the other hand, the right-hand side of (12) represents the slope of the principal's iso-payoff curve. Hence, (12) is a single-crossing condition asserting that the iso-payoff curve always has a steeper slope than (13). To see that this implies complementarity between the two agents' efforts, suppose that $V$ has the CES form: $V(a)=\sum_{t}\left\{\left(a_{t}^{1}\right)^{m}+\left(a_{t}^{2}\right)^{m}\right\}^{1 / m}$, where $m \leq 1$ and $m \neq 0$. In this case, the righthand side of (12) equals $\left(a_{1}^{1} / a_{1}^{2}\right)^{m-1}$. Hence, (12) is easy to satisfy when $m-1$ is negative and large in absolute value. In particular, it will hold for any $c_{1}$ as $m \rightarrow-\infty$, or $V(a)=\sum_{t} \min \left\{a_{t}^{1}, a_{t}^{2}\right\}$ in the limit. On the other hand, in the case of perfect substitutes $m=1$, the inequality reduces to $c_{1}^{\prime \prime}\left(a_{1}^{1}\right)-c_{1}^{\prime \prime}\left(a_{1}^{2}\right)<4 \bar{\phi}^{\prime}\left(a_{1}^{1}-a_{1}^{2}\right)$, which in effect requires $c_{1}^{\prime \prime \prime}$ to be not too negative. The second assumption below requires that the density of the aggregate noise be maximized at the origin.

Assumption 3. $\bar{\phi}(0)=\max _{x \in \mathbf{R}} \bar{\phi}(x)$.

It can be readily verified that Assumption 3 holds if both densities $\phi_{1}$ and $\phi_{2}$ are unimodal at the origin. As seen in the Appendix (Lemma A.2), Assumptions 2 and 3 together guarantee that the principal's payoff is maximized at the symmetric point $\left(a_{1}^{*}, a_{1}^{*}\right)$ along (13) (provided that the stage 2 efforts are symmetric). The next theorem shows that when these conditions hold, the principal's payoff is maximized in the symmetric equilibrium under the no-feedback policy if the stage 2 marginal cost function is convex.

Theorem 4.4. Suppose that Assumptions 1-3 hold. If the marginal cost function $c_{2}^{\prime}$ for stage 2 is convex over $\left[0,\left(c_{2}^{\prime}\right)^{-1}\left(\sup _{x \in \mathbf{R}} \phi_{2}(x)\right)\right]$, then the no-feedback policy maximizes $\bar{v}(\cdot)$ among all policies.

Proof. See the Appendix.

For the other type of the conclusion, we also need the density function of the stage 2 noise to be unimodal.

Assumption 4. $\phi_{2}$ is unimodal at $0: \phi_{2}$ is strictly increasing over $(-\infty, 0)$ and strictly decreasing over $(0, \infty)$.

Under Assumptions 1-4, we obtain the optimality of the full-feedback policy when the stage 2 marginal cost function is concave. 
Theorem 4.5. Suppose that Assumptions 1-4 hold. If the marginal cost function $c_{2}^{\prime}$ for stage 2 is concave over $\left[0,\left(c_{2}^{\prime}\right)^{-1}\left(\phi_{2}(0)\right)\right]$, then the full-feedback policy maximizes $\bar{v}(\cdot)$ among all policies. ${ }^{17}$

Proof. See the Appendix.

As in the case of symmetric PBE, the principal's payoff is independent of the choice of a feedback policy when the stage 2 cost function $c_{2}$ is quadratic.

\section{Discussions}

The paper gives a first attempt to understand the use of the designer's private information in a dynamic tournament, and its conclusion shows that the optimal feedback depends sensitively on the functional form of the agents' disutility of effort. Although the present model abstracts from many important features of real tournaments, we believe that such sensitivity is at the core of the information revelation problem. Below are discussions of some points.

- Principal's commitment:

The most crucial assumption of the present model is that the principal commits to his feedback policy. ${ }^{18}$ The assumption of commitment can be an issue when the principal's private information is largely subjective and not verifiable ex post as in the case of a job tournament where a boss quantifies his impression of his subordinates' performance. The most effective commitment device in such a case is delegation of the work of evaluation and feedback to a division (i.e., a personnel department) or individual whose incentive is not directly affected by the agents' effort. Another effective way is to involve multiple individuals in the evaluation and feedback process so that a report from a single individual can be checked against the reports from other individuals. ${ }^{19}$ The principal's concerns for a reputation can also be a source of his commitment power when the process is repeated over time: If

\footnotetext{
${ }^{17}$ Note that $\sup _{x \in \mathbf{R}} \phi_{2}(x)=\phi_{2}(0)$ under Assumption 4.

${ }^{18}$ Note that commitment by the designer is a standard assumption of the mechanism design literature. In the analysis of auctions, for example, an auctioneer is assumed to retain his good if no bid reaches the reserve price.

${ }^{19}$ Some elements of these can be found in performance assessment of the real world. The multilayered evaluation requires not only the immediate superior of a worker but also the superior's superior to be involved. The 360-degree evaluation requires not only the superior of a worker but also his subordinates, coworkers and customers to be involved.
} 
the announcements over some time span have led to stage 2 efforts whose empirical distribution deviates significantly from the theoretical prediction, then the principal must face the loss of a reputation. ${ }^{20}$

When the principal's commitment is an issue, not all feedback policies are equally credible: The no-feedback policy is the most credible since any release of information is a clear deviation from the rule. Other policies are less credible since commitment can only be checked when the principal's announcement is compared with his private information. Such a variation in credibility levels would be an important consideration when we take the interpretation that a third party is called in to enforce commitment. If the principal totally lacks commitment and optimally chooses his announcement after seeing his signal, then his announcement becomes cheap talk. In this case, if the stage 2 effort were influenced by the announcement, then the message chosen would always be the one that maximizes the effort. However, this implies that the announcement cannot have any informational content. ${ }^{21}$ In other words, the lack of commitment is equivalent to no-feedback in the present framework.

- More than two stages:

The conclusions of the two stage model partially generalize to a $T$-stage tournament. A feedback policy in a $T$-stage model is a contingent plan which determines not only the amount of information revealed but also its timing. For example, the principal may choose to reveal the stage 1 score before stage 3 if the stage 2 score is in some range, but withhold it until stage 4 otherwise. Under a slightly stronger set of assumptions on noise, we can prove that a symmetric PBE exists in the $T$-stage model when a feedback policy is such that $f_{t}\left(x_{1}, \ldots, x_{t-1}\right)=f_{t}\left(-x_{1}, \ldots,-x_{t-1}\right)$ for any stage $t$ score difference $x_{t}=z_{t}^{1}-z_{t}^{2}$ and $t=1, \ldots, T$. Furthermore, among such feedback policies, the no-feedback policy is optimal when the stage $t$ marginal cost functions are convex for $t=2, \ldots, T$, and the feedback policy that reveals the absolute value of the stage score after every period, and hence is "most revealing" in such a class, is optimal when the stage $t$ marginal cost functions are concave. ${ }^{22}$

- Third derivatives:

As seen above, our theory of information feedback highlights the role of the

\footnotetext{
${ }^{20}$ The full analysis of a reputation model, however, is difficult and beyond the scope of this paper.

${ }^{21}$ Kaplan and Zamir (2000) find that the auctioneer cannot exploit his private information on the bidders' valuation if he cannot commit to an announcement policy.

${ }^{22}$ See Aoyagi (2006).
} 
third derivative of the agents' disutility of effort. Lizzeri et al. (2002) also note that the third derivative plays a key role in determining the optimal policies. While economic theory often points out the relevance of the higher-order derivatives in incentive problems, no general consensus exists on their signs. ${ }^{23}$ In this sense, it is premature to discuss implications of the present analysis. However, it is important to realize that optimizing over feedback policies can lead to a substantial improvement in the induced expected effort, and that the optimal information feedback sensitively depends on the underlying parameters. For example, an optimal policy under concave marginal disutility is least desirable under convex marginal disutility, and vice versa.

- Marginal cost that is neither concave nor convex:

One interesting question concerns the optimal feedback policy when the marginal cost of effort is neither convex nor concave on the relevant domain. We provide a partial answer to this question in the supplement by examining the marginal cost function having a single reflection point at which its curvature changes from convex to concave. Our candidate feedback policy reveals full information when the absolute value of the score is less than some threshold, and reveals nothing (other than the fact that the threshold has been exceeded) otherwise. We show that such a feedback policy outperforms the full-feedback policy. A similar argument identifies the circumstance in which no-feedback policy is dominated by the feedback policy that only reveals whether or not the score has exceeded some threshold.

- Private feedback:

In some applications, it is more appropriate to suppose that the agents inherently know their own performance. In a promotion tournament, for example, if the performance of each agent is measured objectively by the number of products they have sold, then each agent learns his own performance even if no information feedback is provided. The only option for the principal in this case is whether to reveal additional information to each agent on the performance of the other agent.

\footnotetext{
${ }^{23}$ For example, Rogerson (1985) shows in a two-stage principal-agent model with public information that a condition involving the third derivative of the agent's utility function determines whether the expected wage should rise or fall over time. The precautionary saving motive in the face of future uncertainty is also characterized by the third derivative of an individual's utility function. See, for example, Kimball (1990). In mechanism design, the optimality of a deterministic mechanism is also associated with the third-order cross derivatives of an agent's utility function. See, for example, Fudenberg and Tirole (1991).
} 
The main difficulty in this problem is the possible asymmetry of the stage 2 efforts caused by asymmetric information. ${ }^{24}$

\section{A Appendix}

\section{A.1 Proofs}

This section collects proofs of the theorems presented in the text. For some of the results, including Theorems 3.1 and 3.2, we do not need to assume that the feedback policy $f$ is a function of $x_{1}$. In the general specification, hence, we let $f: \mathbf{R}^{2} \rightarrow Y$ and denote by $f\left(z_{1}\right)$ the announcement when the stage 1 score profile equals $z_{1}=\left(z_{1}^{1}, z_{1}^{2}\right)$. Just as in the text, denote by $f^{-1}(y) \subset \mathbf{R}^{2}$ the set of stage 1 score profiles $z_{1}=\left(z_{1}^{1}, z_{1}^{2}\right)$ compatible with the announcement $y$. By the regularity assumption, $f^{-1}(y)$ either has positive measure or is countable. For simplicity, the analysis in the Appendix only deals with the case where $f^{-1}(y)$ has positive measure. The treatment of the countable case is similar with the replacement of any integral over $f^{-1}(y)$ by the corresponding summation.

Given a strategy profile $\sigma$ and announcement $y$, let $g_{1}^{\sigma}\left(z_{1} \mid a_{1}^{i}, y\right)$ be the conditional density of the stage 1 score profile $z_{1}$ when the stage 1 effort profile is $\left(a_{1}^{i}, \sigma_{1}^{j}\right)$ (i.e., when agent $i$ chooses a possibly off-equilibrium action $a_{1}^{i}$ while agent $j$ chooses the equilibrium effort level). For $z_{1} \in f^{-1}(y)$, the conditional density can be explicitly written as

$$
\begin{aligned}
& g_{1}^{\sigma}\left(z_{1} \mid a_{1}^{1}, y\right)=\frac{\xi_{1}\left(z_{1}^{1}-a_{1}^{1}, z_{1}^{2}-\sigma_{1}^{2}\right)}{\int_{f^{-1}(y)} \xi_{1}\left(\hat{z}_{1}^{1}-a_{1}^{1}, \hat{z}_{1}^{2}-\sigma_{1}^{2}\right) d \hat{z}_{1}}, \quad \text { and } \\
& g_{1}^{\sigma}\left(z_{1} \mid a_{1}^{2}, y\right)=\frac{\xi_{1}\left(z_{1}^{1}-\sigma_{1}^{1}, z_{1}^{2}-a_{1}^{2}\right)}{\int_{f^{-1}(y)} \xi_{1}\left(\hat{z}_{1}^{1}-\sigma_{1}^{1}, \hat{z}_{1}^{2}-a_{1}^{2}\right) d \hat{z}_{1}} .
\end{aligned}
$$

Note in particular that $g_{1}^{\sigma}\left(\cdot \mid a_{1}^{i}, y\right)$ depends on $\sigma$ only through the stage 1 profile $\sigma_{1}$. With slight abuse of notation, we define $g_{1}^{\sigma}\left(z_{1} \mid y\right)=g_{1}^{\sigma}\left(z_{1} \mid \sigma_{1}^{i}, y\right)$ : the density of $z_{1}$ conditional on $y$ when both agents choose their effort according to $\sigma$. Its explicit form is given by

$$
g_{1}^{\sigma}\left(z_{1} \mid y\right)=\frac{\xi_{1}\left(z_{1}-\sigma_{1}\right)}{\int_{f^{-1}(y)} \xi_{1}\left(\hat{z}_{1}-\sigma_{1}\right) d \hat{z}_{1}} .
$$

\footnotetext{
${ }^{24}$ Analysis of private feedback under the simplifying assumption that the performance noise in stage 1 can take only one of two values is available at http://www.eonet.ne.jp/ ${ }^{\sim}$ aoyagi/ private_feedback.pdf.
} 
Proof of Theorem 3.1 We first state the first-order conditions (7) for the case where $f$ is a function of $z_{1}$ :

$$
\begin{aligned}
& c_{1}^{\prime}\left(\sigma_{1}^{1}\right)=\bar{\phi}\left(\sigma_{1}^{1}-\sigma_{1}^{2}\right)+\int_{\mathbf{R}^{2}} c_{2}\left(a_{2}^{*}\left(\sigma_{1}, f\left(z_{1}\right)\right)\right) \frac{\partial \xi_{1}}{\partial \varepsilon_{1}^{1}}\left(z_{1}-\sigma_{1}\right) d z_{1}, \\
& c_{1}^{\prime}\left(\sigma_{1}^{2}\right)=\bar{\phi}\left(\sigma_{1}^{1}-\sigma_{1}^{2}\right)+\int_{\mathbf{R}^{2}} c_{2}\left(a_{2}^{*}\left(\sigma_{1}, f\left(z_{1}\right)\right)\right) \frac{\partial \xi_{1}}{\partial \varepsilon_{1}^{2}}\left(z_{1}-\sigma_{1}\right) d z_{1} .
\end{aligned}
$$

A change of variables from $z_{1}=\left(z_{1}^{1}, z_{1}^{2}\right)$ to $\left(x_{1}, s_{1}\right)=\left(z_{1}^{1}-z_{1}^{2}, z_{1}^{1}+z_{1}^{2}\right)$ shows that (14) reduces to (7) when $f$ is a function of $x_{1}$ alone.

Fix any PBE $\sigma$. Recall that $\pi_{2}^{i}\left(a_{2}^{i} \mid \sigma, a_{1}^{i}, y\right)$ represents agent $i$ 's expected payoff in stage 2 when he chooses $a_{2}^{i}$ in stage 2 , his history after stage 1 is $h_{1}^{i}=\left(a_{1}^{i}, y\right)$, and agent $j$ plays according to the equilibrium strategy $\sigma^{j}$. For simplicity, write $\pi_{2}^{i}\left(a_{2}^{i} \mid a_{1}^{i}, y\right)$ for $\pi_{2}^{i}\left(a_{2}^{i} \mid \sigma, a_{1}^{i}, y\right)$. As seen in the text, we have

$$
\frac{\partial \pi_{2}^{1}}{\partial a_{2}^{1}}\left(a_{2}^{1} \mid a_{1}^{1}, y\right)=E_{z_{1}}\left[\phi_{2}\left(a_{2}^{1}-\sigma_{2}^{2}(y)+x_{1}\right) \mid a_{1}^{1}, y\right]-c_{2}^{\prime}\left(a_{2}^{1}\right) .
$$

Since $\frac{\partial \pi_{2}^{1}}{\partial a_{2}^{1}}\left(0 \mid a_{1}^{1}, y\right)>0$ by $c_{2}^{\prime}(0)=0$, the sequentially rational action $\sigma_{2}^{1}\left(a_{1}^{1}, y\right)$ in stage 2 (if any) must satisfy the FOC

$$
c_{2}^{\prime}\left(\sigma_{2}^{1}\left(a_{1}^{1}, y\right)\right)=E_{z_{1}}\left[\phi_{2}\left(\sigma_{2}^{1}\left(a_{1}^{1}, y\right)-\sigma_{2}^{2}(y)+x_{1}\right) \mid a_{1}^{1}, y\right]
$$

for every $a_{1}^{1}$. Since $\inf _{a \in \mathbf{R}_{+}} c_{2}^{\prime \prime}(a)>\sup _{x \in \mathbf{R}} \phi_{2}^{\prime}(x)$ by assumption, we also have $\frac{\partial^{2} \pi_{2}^{1}}{\partial\left(a_{2}^{1}\right)^{2}}\left(a_{2}^{1} \mid a_{1}^{1}, y\right)<0$. It then follows that the above FOC is indeed sufficient for global maximization, and also that $\sigma_{2}^{1}\left(a_{1}^{1}, y\right)$ is differentiable as a function of $a_{1}^{1}$ by the implicit function theorem. Likewise, agent 2's stage 2 action satisfies

$$
c_{2}^{\prime}\left(\sigma_{2}^{2}\left(a_{1}^{2}, y\right)\right)=E_{z_{1}}\left[\phi_{2}\left(-\sigma_{2}^{1}(y)+\sigma_{2}^{2}\left(a_{1}^{2}, y\right)-x_{1}\right) \mid a_{1}^{2}, y\right]
$$

for every $a_{1}^{2}$. On the equilibrium path where $a_{1}^{i}=\sigma_{1}^{i}$, we have $\sigma_{2}^{i}\left(\sigma_{1}^{i}, y\right)=\sigma_{2}^{i}(y)$ and $E_{z_{1}}\left[\cdot \mid \sigma_{1}^{i}, y\right]=E_{z_{1}}[\cdot \mid y]$. Hence, (15) and (16) show that $\sigma_{2}^{1}(y)$ and $\sigma_{2}^{2}(y)$ must satisfy

$$
\sigma_{2}^{1}(y)=\sigma_{2}^{2}(y)=a_{2}^{*}(y) \equiv\left(c_{2}^{\prime}\right)^{-1}\left(E_{z_{1}}\left[\phi_{2}\left(x_{1}\right) \mid y\right]\right) .
$$

Now let $\pi_{1}^{i}\left(a_{1}^{i}\right)=\pi_{1}^{i}\left(a_{1}^{i} \mid \sigma\right)$ be agent $i$ 's (overall) expected payoff when he takes $a_{1}^{i}$ in stage 1 and $\sigma_{2}^{i}\left(a_{1}^{i}, y\right)$ in stage 2 , while agent $j$ plays according to his equilibrium strategy $\sigma^{j}$ :

$$
\begin{gathered}
\pi_{1}^{1}\left(a_{1}^{1}\right)=\int_{\mathbf{R}^{2}}\left\{\Phi_{2}\left(\sigma_{2}^{1}\left(a_{1}^{1}, f\left(z_{1}\right)\right)-\sigma_{2}^{2}\left(f\left(z_{1}\right)\right)+x_{1}\right)-c_{2}\left(\sigma_{2}^{1}\left(a_{1}^{1}, f\left(z_{1}\right)\right)\right)\right\} \\
\times \xi_{1}\left(z_{1}^{1}-a_{1}^{1}, z_{1}^{2}-\sigma_{1}^{2}\right) d z_{1}-c_{1}\left(a_{1}^{1}\right)
\end{gathered}
$$


and

$$
\begin{gathered}
\pi_{1}^{2}\left(a_{1}^{2}\right)=\int_{\mathbf{R}^{2}}\left\{\Phi_{2}\left(\sigma_{2}^{2}\left(a_{1}^{2}, f\left(z_{1}\right)\right)-\sigma_{2}^{1}\left(f\left(z_{1}\right)\right)-x_{1}\right)-c_{2}\left(\sigma_{2}^{2}\left(a_{1}^{2}, f\left(z_{1}\right)\right)\right)\right\} \\
\times \xi_{1}\left(z_{1}^{1}-\sigma_{1}^{1}, z_{1}^{2}-a_{1}^{2}\right) d z_{1}-c_{1}\left(a_{1}^{2}\right)
\end{gathered}
$$

Given that $\sigma_{2}^{1}$ is differentiable in $a_{1}^{1}$ as noted above, we use the envelope theorem to differentiate $\pi_{1}^{1}$ :

$$
\begin{aligned}
& \left(\pi_{1}^{1}\right)^{\prime}\left(a_{1}^{1}\right) \\
& =-\int_{\mathbf{R}^{2}} \Phi_{2}\left(\sigma_{2}^{1}\left(a_{1}^{1}, f\left(z_{1}\right)\right)-\sigma_{2}^{2}\left(f\left(z_{1}\right)\right)+x_{1}\right) \frac{\partial \xi_{1}}{\partial \varepsilon_{1}^{1}}\left(z_{1}^{1}-a_{1}^{1}, z_{1}^{2}-\sigma_{1}^{2}\right) d z_{1} \\
& +\int_{\mathbf{R}^{2}} c_{2}\left(\sigma_{2}^{1}\left(a_{1}^{1}, f\left(z_{1}\right)\right)\right) \frac{\partial \xi_{1}}{\partial \varepsilon_{1}^{1}}\left(z_{1}^{1}-a_{1}^{1}, z_{1}^{2}-\sigma_{1}^{2}\right) d z_{1}-c_{1}^{\prime}\left(a_{1}^{1}\right) .
\end{aligned}
$$

If the equilibrium stage 1 action $a_{1}^{1}=\sigma_{1}^{1}$ is strictly positive, the FOC $\left(\pi_{1}^{1}\right)^{\prime}\left(\sigma_{1}^{1}\right)=0$ must hold. Since $\sigma_{2}^{1}(y)=\sigma_{2}^{2}(y)$ for any $y \in Y$ by (17), this FOC is equivalent to

$$
c_{1}^{\prime}\left(\sigma_{1}^{1}\right)=\int_{\mathbf{R}^{2}}\left\{-\Phi_{2}\left(x_{1}\right)+c_{2}\left(a_{2}^{*}\left(\sigma_{1}, f\left(z_{1}\right)\right)\right)\right\} \frac{\partial \xi_{1}}{\partial \varepsilon_{1}^{1}}\left(z_{1}-a_{1}\right) d z_{1} .
$$

Changing variables of the first integrand, and then integrating it by parts, we see that this is equivalent to the first line of (14). The symmetric argument shows that the second line of (14) is equivalent to the FOC for agent 2.

Proof of Theorem 3.2 Write $\varepsilon=\kappa / 2$, where $\kappa$ is as defined in Assumption 1 . Suppose that $\sigma_{1}=\left(\sigma_{1}^{1}, \sigma_{1}^{2}\right)$ solves (14). We construct a PBE as follows. First, for each $a_{1}^{1}, a_{1}^{2} \in \mathbf{R}$, and $y \in Y$, let

$$
\varphi_{2}^{1}\left(a_{2}^{1} \mid a_{1}^{1}, y\right)=E_{z_{1}}\left[\phi_{2}\left(a_{2}^{1}-a_{2}^{*}\left(\sigma_{1}, y\right)+x_{1}\right) \mid a_{1}^{1}, y\right]-c_{2}^{\prime}\left(a_{2}^{1}\right),
$$

and

$$
\varphi_{2}^{2}\left(a_{2}^{2} \mid a_{1}^{2}, y\right)=E_{z_{1}}\left[\phi_{2}\left(a_{2}^{*}\left(\sigma_{1}, y\right)-a_{2}^{2}+x_{1}\right) \mid a_{1}^{2}, y\right]-c_{2}^{\prime}\left(a_{2}^{2}\right) .
$$

Define $\sigma_{2}^{1}\left(a_{1}^{1}, y\right)>0$ and $\sigma_{2}^{2}\left(a_{1}^{2}, y\right)>0$ to be the unique solutions to

$$
\varphi_{2}^{1}\left(a_{2}^{1} \mid a_{1}^{1}, y\right)=0 \quad \text { and } \quad \varphi_{2}^{2}\left(a_{2}^{2} \mid a_{1}^{2}, y\right)=0,
$$

respectively. To see that such a solution exists, note that $\varphi_{2}^{1}\left(0 \mid a_{1}^{1}, y\right)>0$ since $c_{2}^{\prime}(0)=0$ and $\phi_{2}>0$, and that $\varphi_{2}^{1}\left(a_{2}^{1} \mid a_{1}^{1}, y\right)<0$ for $a_{2}^{1}$ large enough since 
$\lim _{a \rightarrow \infty} c_{2}^{\prime}(a)>\varepsilon>\sup _{x \in \mathbf{R}} \phi_{2}(x)$. Furthermore, it follows from $\inf _{a \in \mathbf{R}} c_{2}^{\prime \prime}(a)>$ $\varepsilon>\sup _{x \in \mathbf{R}} \phi_{2}^{\prime}(x)$ that

$$
\begin{aligned}
\frac{\partial \varphi_{2}^{1}}{\partial a_{2}^{1}}\left(a_{2}^{1} \mid a_{1}^{1}, y\right) & =-c_{2}^{\prime \prime}\left(a_{2}^{1}\right)+E_{z_{1}}\left[\phi_{2}^{\prime}\left(a_{2}^{1}-\sigma_{2}^{2}(y)+x_{1}\right) \mid a_{1}^{1}, y\right] \\
& <-\kappa+\varepsilon<0 .
\end{aligned}
$$

Hence there indeed exists a unique solution $\sigma_{2}^{1}\left(a_{1}^{1}, y\right)>0$ to $\varphi_{2}^{1}\left(a_{2}^{1} \mid a_{1}^{1}, y\right)=0$. The symmetric argument applies to agent 2 . Note now that when $a_{1}^{i}=\sigma_{1}^{i}, a_{2}^{i}=$ $a_{2}^{*}\left(\sigma_{1}, y\right)$ solves $\varphi_{2}^{i}\left(a_{2}^{i} \mid \sigma_{1}^{i}, y\right)=0$. We can hence replace $a_{2}^{*}\left(\sigma_{1}, y\right)$ in the definition of $\varphi_{2}^{1}\left(a_{2}^{1} \mid a_{1}^{1}, y\right)$ by $\sigma_{2}^{2}(y)=\sigma_{2}^{2}\left(\sigma_{1}^{2}, y\right)$, and see that $\varphi_{2}^{1}\left(a_{2}^{1} \mid a_{1}^{1}, y\right)=0$ is equivalent to the FOC $\frac{\partial \pi_{2}^{1}}{\partial a_{2}^{1}}\left(a_{2}^{1} \mid a_{1}^{1}, y\right)=0$ ((15) in the proof of Theorem 1) of agent 1's stage 2 payoff maximization problem. To see that $a_{2}^{1}=\sigma_{2}^{1}\left(a_{1}^{1}, y\right)$ is sequentially rational, note that it is a global maximizer of $\pi_{2}^{1}$ since

$$
\begin{aligned}
\frac{\partial^{2} \pi_{2}^{1}}{\partial\left(a_{2}^{1}\right)^{2}}\left(a_{2}^{1} \mid a_{1}^{1}, y\right) & =-c_{2}^{\prime \prime}\left(a_{2}^{1}\right)+E_{z_{1}}\left[\phi_{2}^{\prime}\left(a_{2}^{1}-\sigma_{2}^{2}(y)+x_{1}\right) \mid a_{1}^{1}, y\right] \\
& <-\kappa+\varepsilon<0 .
\end{aligned}
$$

The same observation holds for agent 2 .

We now turn to the analysis of stage 1 action. As in the proof of Theorem 3.1, write $\pi_{1}^{i}\left(a_{1}^{i}\right)=\pi_{1}^{i}\left(a_{1}^{i} \mid \sigma\right)$ for agent $i$ 's overall payoff when he chooses $a_{1}^{i}$ in stage 1 and $\sigma_{2}^{i}\left(a_{1}^{i}, y\right)$ in stage 2 , and agent $j$ plays according to $\sigma^{2}$. Given $\sigma_{2}^{i}\left(a_{1}^{i}, y\right)$ defined above, let for $i=1,2$,

$$
\varphi_{1}^{i}\left(a_{1}^{i}\right)=\left(\pi_{1}^{i}\right)^{\prime}\left(a_{1}^{i}\right)
$$

where the explicit form of the right-hand side is as in (19) in the proof of Theorem 3.1. Since (19) is identical to the left-hand sides of (14) when $a_{1}^{i}=\sigma_{1}^{i}, \varphi_{1}^{i}\left(\sigma_{1}^{i}\right)=0$ holds by assumption, and $\sigma_{1}^{i}$ is a solution to the FOC of agent $i$ 's payoff maximization problem. In what follows, we will show that $\sigma_{1}^{i}$ is indeed the global maximizer of $\pi_{1}^{1}$ by demonstrating $\left(\varphi_{1}^{i}\right)^{\prime}=\left(\pi_{1}^{i}\right)^{\prime \prime}<0$.

Since $\sigma_{2}^{1}$ is differentiable with respect to $a_{1}^{1}$ as noted in the proof of Theorem 
3.1, we can differentiate (19) to obtain

$$
\begin{gathered}
\left(\varphi_{1}^{1}\right)^{\prime}\left(a_{1}^{1}\right)=-c_{1}^{\prime \prime}\left(a_{1}^{1}\right) \\
-\int_{\mathbf{R}^{2}}\left\{\phi_{2}\left(\sigma_{2}^{1}\left(a_{1}^{1}, f\left(z_{1}\right)\right)-\sigma_{2}^{2}\left(f\left(z_{1}\right)\right)+x_{1}\right)-c_{2}^{\prime}\left(\sigma_{2}^{1}\left(a_{1}^{1}, f\left(z_{1}\right)\right)\right)\right\} \\
\times \frac{\partial \sigma_{2}^{1}}{\partial a_{1}^{1}}\left(a_{1}^{1}, f\left(z_{1}\right)\right) \frac{\partial \xi_{1}}{\partial \varepsilon_{1}^{1}}\left(z_{1}^{1}-a_{1}^{1}, z_{1}^{2}-\sigma_{1}^{2}\right) d z_{1} \\
+\int_{\mathbf{R}^{2}}\left\{\Phi_{2}\left(\sigma_{2}^{1}\left(a_{1}^{1}, f\left(z_{1}\right)\right)-\sigma_{2}^{2}\left(f\left(z_{1}\right)\right)+x_{1}\right)-c_{2}\left(\sigma_{2}^{1}\left(a_{1}^{1}, f\left(z_{1}\right)\right)\right)\right\} \\
\times \frac{\partial^{2} \xi_{1}}{\partial\left(\varepsilon_{1}^{1}\right)^{2}}\left(z_{1}^{1}-a_{1}^{1}, z_{1}^{2}-\sigma_{1}^{2}\right) d z_{1} .
\end{gathered}
$$

Note now that for any $y \in Y$, we have $c_{2}^{\prime}\left(\sigma_{2}^{1}\left(a_{1}^{1}, y\right)\right) \leq \varepsilon$ by $(16)$ and $c_{2}\left(\sigma_{2}^{1}\left(a_{1}^{1}, y\right)\right) \leq 1$ by the above observation that $\sigma_{2}^{1}\left(a_{1}^{1}, y\right)$ maximizes $\pi_{2}^{1}\left(\cdot \mid a_{1}^{1}, y\right)$. Hence,

$$
\left|\phi_{2}\left(\sigma_{2}^{1}\left(a_{1}^{1}, f\left(z_{1}\right)\right)-\sigma_{2}^{2}\left(f\left(z_{1}\right)\right)+x_{1}\right)-c_{2}^{\prime}\left(\sigma_{2}^{1}\left(a_{1}^{1}, f\left(z_{1}\right)\right)\right)\right| \leq \varepsilon,
$$

and

$$
\left|\Phi_{2}\left(\sigma_{2}^{1}\left(a_{1}^{1}, f\left(z_{1}\right)\right)-\sigma_{2}^{2}\left(f\left(z_{1}\right)\right)+x_{1}\right)-c_{2}\left(\sigma_{2}^{1}\left(a_{1}^{1}, f\left(z_{1}\right)\right)\right)\right| \leq 1 .
$$

It follows that

$$
\begin{aligned}
\left(\varphi_{1}^{1}\right)^{\prime}\left(a_{1}^{1}\right) & \leq-c_{1}^{\prime \prime}\left(a_{1}^{1}\right)+\varepsilon \int_{\mathbf{R}^{2}}\left|\frac{\partial \sigma_{2}^{1}}{\partial a_{1}^{1}}\left(a_{1}^{1}, f\left(z_{1}\right)\right)\right|\left|\frac{\partial \xi_{1}}{\partial \varepsilon_{1}^{1}}\left(z_{1}^{1}-a_{1}^{1}, z_{1}^{2}-\sigma_{1}^{2}\right)\right| d z_{1} \\
& +\int_{\mathbf{R}^{2}}\left|\frac{\partial^{2} \xi_{1}}{\partial\left(\varepsilon_{1}^{1}\right)^{2}}\left(z_{1}^{1}-a_{1}^{1}, z_{1}^{2}-\sigma_{1}^{2}\right)\right| d z_{1} .
\end{aligned}
$$

For simplicity, write $\hat{E}_{z_{1}}$ for $E_{z_{1}}\left[\cdot \mid a_{1}^{1}\right]$, expectation given the stage 1 effort profile $\left(a_{1}^{1}, \sigma_{1}^{2}\right)$. Define

$$
q\left(z_{1}\right)=\frac{\left|\frac{\partial \xi_{1}}{\partial \varepsilon_{1}^{1}}\left(z_{1}^{1}-a_{1}^{1}, z_{1}^{2}-\sigma_{1}^{2}\right)\right|}{\xi_{1}\left(z_{1}^{1}-a_{1}^{1}, z_{1}^{2}-\sigma_{1}^{2}\right)} .
$$

By Lemma A.1 below, the integral in the second term on the right-hand side of (21) is evaluated as

$$
\begin{aligned}
& \int_{\mathbf{R}^{2}}\left|\frac{\partial \sigma_{2}^{1}}{\partial a_{1}^{1}}\left(a_{1}^{1}, f\left(z_{1}\right)\right)\right|\left|\frac{\partial \xi_{1}}{\partial \varepsilon_{1}^{1}}\left(z_{1}^{1}-a_{1}^{1}, z_{1}^{2}-\sigma_{1}^{2}\right)\right| d z_{1} \\
& <\frac{2 \varepsilon}{\kappa-\varepsilon} \int_{\mathbf{R}^{2}} \hat{E}_{\hat{z}_{1}}\left[q\left(\hat{z}_{1}\right) \mid f\left(\hat{z}_{1}\right)=f\left(z_{1}\right)\right]\left|\frac{\partial \xi_{1}}{\partial \varepsilon_{1}^{1}}\left(z_{1}^{1}-a_{1}^{1}, z_{1}^{2}-\sigma_{1}^{2}\right)\right| d z_{1} .
\end{aligned}
$$


Note now that the above integral can further be rewritten as

$$
\begin{aligned}
& \int_{\mathbf{R}^{2}} \hat{E}_{\hat{z}_{1}}\left[q\left(\hat{z}_{1}\right) \mid f\left(\hat{z}_{1}\right)=f\left(z_{1}\right)\right] q\left(z_{1}\right) \xi_{1}\left(z_{1}^{1}-a_{1}^{1}, z_{1}^{2}-\sigma_{1}^{2}\right) d z_{1} \\
& =\hat{E}_{z_{1}}\left[\hat{E}_{\hat{z}_{1}}\left[q\left(\hat{z}_{1}\right) \mid f\left(\hat{z}_{1}\right)=f\left(z_{1}\right)\right] q\left(z_{1}\right)\right] \\
& \leq \hat{E}_{z_{1}}\left[\hat{E}_{\hat{z}_{1}}\left[q\left(\hat{z}_{1}\right) \mid f\left(\hat{z}_{1}\right)=f\left(z_{1}\right)\right]^{2}\right]^{1 / 2} \hat{E}_{z_{1}}\left[q\left(z_{1}\right)^{2}\right]^{1 / 2} \\
& \leq \hat{E}_{z_{1}}\left[\hat{E}_{\hat{z}_{1}}\left[q\left(\hat{z}_{1}\right)^{2} \mid f\left(\hat{z}_{1}\right)=f\left(z_{1}\right)\right]\right]^{1 / 2} \hat{E}_{z_{1}}\left[q\left(z_{1}\right)^{2}\right]^{1 / 2} \\
& =\hat{E}_{z_{1}}\left[q\left(z_{1}\right)^{2}\right]=\int_{\mathbf{R}^{2}} \frac{\frac{\partial \xi_{1}}{\partial \varepsilon_{1}^{1}}\left(z_{1}^{1}-a_{1}^{1}, z_{1}^{1}-\sigma_{1}^{2}\right)^{2}}{\xi_{1}\left(z_{1}^{1}-a_{1}^{1}, z_{1}^{2}-\sigma_{1}^{2}\right)} d z_{1}<\varepsilon
\end{aligned}
$$

where the third line follows from Schwartz' inequality, the fourth line from Jensen's inequality, and the last inequality from Assumption 1. It follows that we can evaluate the right-hand side of (21) as

$$
\left(\varphi_{1}^{1}\right)^{\prime}\left(a_{1}^{1}\right)<-\kappa+\frac{2 \varepsilon^{3}}{\kappa-\varepsilon}+\varepsilon \leq 0 .
$$

This proves the claim.

\section{Lemma A.1.}

$$
\left|\frac{\partial \sigma_{2}^{1}}{\partial a_{1}^{1}}\left(a_{1}^{1}, y\right)\right|<\frac{2 \varepsilon}{\kappa-\varepsilon} \hat{E}_{z_{1}}\left[q\left(z_{1}\right) \mid y\right] .
$$

Proof. For $z_{1} \in f^{-1}(y)$, we have

$$
\begin{aligned}
\frac{\partial g_{1}^{\sigma}}{\partial a_{1}^{1}}\left(z_{1} \mid a_{1}^{1}, y\right) & =\frac{-\frac{\partial \xi_{1}}{\partial \varepsilon_{1}^{1}}\left(z_{1}^{1}-a_{1}^{1}, z_{1}^{2}-\sigma_{1}^{2}\right)}{\int_{f^{-1}(y)} \xi_{1}\left(\hat{z}_{1}^{1}-a_{1}^{1}, \hat{z}_{1}^{2}-\sigma_{1}^{2}\right) d \hat{z}_{1}} \\
& +\frac{\xi_{1}\left(z_{1}^{1}-a_{1}^{1}, z_{1}^{2}-\sigma_{1}^{2}\right) \int_{f^{-1}(y)}\left|\frac{\partial \xi_{1}}{\partial \varepsilon_{1}^{1}}\left(\hat{z}_{1}^{1}-a_{1}^{1}, \hat{z}_{1}^{2}-\sigma_{1}^{2}\right)\right| d \hat{z}_{1}}{\left\{\int_{f^{-1}(y)} \xi_{1}\left(\hat{z}_{1}^{1}-a_{1}^{1}, \hat{z}_{1}^{2}-\sigma_{1}^{2}\right) d \hat{z}_{1}\right\}^{2}}
\end{aligned}
$$

and hence

$$
\begin{aligned}
\int_{\mathbf{R}^{2}}\left|\frac{\partial g_{1}^{\sigma}}{\partial a_{1}^{1}}\left(z_{1} \mid a_{1}^{1}, y\right)\right| d z_{1} & \leq 2 \frac{\int_{f^{-1}(y)}\left|\frac{\partial \xi_{1}}{\partial \varepsilon_{1}^{1}}\left(z_{1}^{1}-a_{1}^{1}, z_{1}^{2}-\sigma_{1}^{2}\right)\right| d z_{1}}{\int_{f^{-1}(y)} \xi_{1}\left(z_{1}^{1}-a_{1}^{1}, z_{1}^{2}-\sigma_{1}^{2}\right) d z_{1}} \\
& =2 \hat{E}_{z_{1}}\left[q\left(z_{1}\right) \mid y\right] .
\end{aligned}
$$


On the other hand,

$$
\frac{\partial \varphi_{2}^{1}}{\partial a_{1}^{1}}\left(a_{2}^{1} \mid a_{1}^{1}, y\right)=\int_{\mathbf{R}^{2}} \phi_{2}\left(a_{2}^{1}-\sigma_{2}^{2}(y)+x_{1}\right) \frac{\partial g_{1}^{\sigma}}{\partial a_{1}^{1}}\left(z_{1} \mid a_{1}^{1}, y\right) d z_{1},
$$

so that

$$
\left|\frac{\partial \varphi_{2}^{1}}{\partial a_{1}^{1}}\left(a_{2}^{1} \mid a_{1}^{1}, y\right)\right|<\varepsilon \int_{\mathbf{R}^{2}}\left|\frac{\partial g_{1}^{\sigma}}{\partial a_{1}^{1}}\left(z_{1} \mid a_{1}^{1}, y\right)\right| d z_{1} \leq 2 \varepsilon \hat{E}_{z_{1}}\left[q\left(z_{1}\right) \mid y\right] .
$$

It then follows from the implicit function theorem and (20) that

$$
\left|\frac{\partial \sigma_{2}^{1}}{\partial a_{1}^{1}}\left(a_{1}^{1}, y\right)\right|=\frac{\left|\frac{\partial \varphi_{2}^{1}}{\partial a_{1}^{1}}\left(\sigma_{2}^{1}\left(a_{1}^{1}, y\right) \mid a_{1}^{1}, y\right)\right|}{\left|\frac{\partial \varphi_{2}^{1}}{\partial a_{2}^{1}}\left(\sigma_{2}^{1}\left(a_{1}^{1}, y\right) \mid a_{1}^{1}, y\right)\right|}<\frac{2 \varepsilon}{\kappa-\varepsilon} \hat{E}_{z_{1}}\left[q\left(z_{1}\right) \mid y\right] .
$$

Proof of Theorem 3.3 Suppose that $\sigma_{1}^{1}=\sigma_{1}^{2}$. We first show that $a_{2}^{*}\left(\sigma_{1}, f\left(x_{1}\right)\right)=$ $a_{2}^{*}\left(\sigma_{1}, f\left(-x_{1}\right)\right)$ for any $x_{1} \in \mathbf{R}$. Since $f$ is symmetric, $g_{1}^{\sigma}\left(\tilde{x}_{1} \mid f\left(x_{1}\right)\right)=g_{1}^{\sigma}\left(-\tilde{x}_{1} \mid\right.$ $\left.f\left(-x_{1}\right)\right)$ for any $\tilde{x}_{1} \in \mathbf{R}$. This and the symmetry of $\phi_{2}$ imply that

$$
\begin{aligned}
a_{2}^{*}\left(\sigma_{1}, f\left(x_{1}\right)\right) & =\left(c_{2}^{\prime}\right)^{-1}\left(\int_{\mathbf{R}} \phi_{2}\left(\tilde{x}_{1}\right) g_{1}^{\sigma}\left(\tilde{x}_{1} \mid f\left(x_{1}\right)\right) d \tilde{x}_{1}\right) \\
& =\left(c_{2}^{\prime}\right)^{-1}\left(\int_{\mathbf{R}} \phi_{2}\left(-\tilde{x}_{1}\right) g_{1}^{\sigma}\left(-\tilde{x}_{1} \mid f\left(x_{1}\right)\right) d \tilde{x}_{1}\right) \\
& =\left(c_{2}^{\prime}\right)^{-1}\left(\int_{\mathbf{R}} \phi_{2}\left(\tilde{x}_{1}\right) g_{1}^{\sigma}\left(\tilde{x}_{1} \mid f\left(-x_{1}\right)\right) d \tilde{x}_{1}\right) \\
& =a_{2}^{*}\left(\sigma_{1}, f\left(-x_{1}\right)\right) .
\end{aligned}
$$

With this equality, $\sigma_{1}^{1}=\sigma_{1}^{2}=a_{1}^{*}$ solves (14) since $\phi_{1}^{\prime}\left(-x_{1}\right)=-\phi_{1}^{\prime}\left(x_{1}\right)$ and hence

$$
\begin{aligned}
& \int_{\mathbf{R}} c_{2}\left(a_{2}^{*}\left(\sigma_{1}, f\left(x_{1}\right)\right)\right) \phi_{1}^{\prime}\left(x_{1}\right) d x_{1} \\
& =\int_{0}^{\infty} c_{2}\left(a_{2}^{*}\left(\sigma_{1}, f\left(x_{1}\right)\right)\right) \phi_{1}^{\prime}\left(x_{1}\right) d x_{1}+\int_{-\infty}^{0} c_{2}\left(a_{2}^{*}\left(\sigma_{1}, f\left(x_{1}\right)\right)\right) \phi_{1}^{\prime}\left(x_{1}\right) d x_{1} \\
& =\int_{0}^{\infty} c_{2}\left(a_{2}^{*}\left(\sigma_{1}, f\left(x_{1}\right)\right)\right) \phi_{1}^{\prime}\left(x_{1}\right) d x_{1}-\int_{0}^{\infty} c_{2}\left(a_{2}^{*}\left(\sigma_{1}, f\left(-x_{1}\right)\right)\right) \phi_{1}^{\prime}\left(x_{1}\right) d x_{1} \\
& =0
\end{aligned}
$$

This completes the proof. 
Lemma A.2. Suppose that Assumptions 2 and 3 hold and that $\lim _{a \rightarrow \infty} c_{1}^{\prime}(a)>$ $2 \bar{\phi}(0)$ for $i=1$, 2. Then for any $\sigma_{1}$ that solves (14) and any $a_{2}$ such that $a_{2}^{1}=a_{2}^{2}$, the principal's payoff function satisfies

$$
V\left(\left(a_{1}^{*}, a_{1}^{*}\right), a_{2}\right) \geq V\left(\sigma_{1}, a_{2}\right) .
$$

Proof. Fix $a_{2} \in \mathbf{R}_{+}^{2}$ such that $a_{2}^{1}=a_{2}^{2}$. Since $h$ is continuous, the inverse image $h^{-1}(\{0\})$ is closed. Furthermore, it is non-empty since $\left(a_{1}^{*}, a_{1}^{*}\right) \in h^{-1}(\{0\})$. To see that it is bounded, note that $\left(c_{1}^{\prime}\right)^{-1}(2 \bar{\phi}(0))<\infty$ by assumption. For any $a_{1}$ such that $\max \left\{a_{1}^{1}, a_{1}^{2}\right\}>\left(c_{1}^{\prime}\right)^{-1}(2 \bar{\phi}(0))$, we have

$$
h\left(a_{1}\right) \geq c_{1}^{\prime}\left(a_{1}^{1}\right)+c_{1}^{\prime}\left(a_{1}^{2}\right)-2 \bar{\phi}(0)>0,
$$

where the first inequality follows from Assumption 3 and the second from the monotonicity of $c_{1}^{\prime}$. This shows that $h^{-1}(\{0\})$ is a subset of the bounded set $\left\{a_{1}: \max \left\{a_{1}^{1}, a_{1}^{2}\right\} \leq\left(c_{1}^{\prime}\right)^{-1}(2 \bar{\phi}(0))\right\}$ and hence is compact. It follows that the continuous function $V\left(\cdot, a_{2}\right)$ on the compact set $h^{-1}(\{0\})=\left\{a_{1} \in \mathbf{R}_{+}^{2}: h\left(a_{1}\right)=0\right\}$ achieves a maximum. Let $\bar{a}_{1}=\left(\bar{a}_{1}^{1}, \bar{a}_{1}^{2}\right) \in h^{-1}(\{0\})$ be any maximizer of $V\left(\cdot, a_{2}\right)$ in $h^{-1}(\{0\})$. We show that $\bar{a}_{1}=\left(a_{1}^{*}, a_{1}^{*}\right)$. Suppose that $\bar{a}_{1}^{1}<\bar{a}_{1}^{2}$. Since $\frac{\partial h}{\partial a_{1}^{2}} \neq 0$ by (12), the implicit function theorem shows that there exists a function $\gamma$ defined in a neighborhood of $\bar{a}_{1}^{1}$ such that $h\left(a_{1}^{1}, \gamma\left(a_{1}^{1}\right)\right)=0$. Furthermore, $\gamma$ is differentiable at $\bar{a}_{1}^{1}$ and the derivative $\gamma^{\prime}\left(\bar{a}_{1}^{1}\right)$ is given by the left-hand side of (12) with $\bar{a}_{1}^{i}$ replacing $a_{1}^{i}$. Now let $\delta\left(a_{1}^{1}\right)=V\left(\left(a_{1}^{1}, \gamma\left(a_{1}^{1}\right)\right), a_{2}\right)$. $\delta$ is also differentiable at $\bar{a}_{1}^{1}$ and its derivative is given by

$$
\delta^{\prime}\left(\bar{a}_{1}^{1}\right)=\frac{\partial V}{\partial a_{1}^{1}}\left(\bar{a}_{1}, a_{2}\right)+\frac{\partial V}{\partial a_{1}^{2}}\left(\bar{a}_{1}, a_{2}\right) \gamma^{\prime}\left(\bar{a}_{1}^{1}\right) .
$$

It can be readily verified that Assumption 2 implies $\delta^{\prime}\left(\bar{a}_{1}^{1}\right)>0$. This contradicts our assumption that $V$ is maximized at $\bar{a}_{1}$ in $h^{-1}(\{0\})=0$. The symmetric argument shows that it cannot be maximized at $\bar{a}$ such that $\bar{a}_{1}^{1}>\bar{a}_{1}^{2}$ either. Hence, we must have $\bar{a}_{1}^{1}=\bar{a}_{1}^{2}=a_{1}^{*}$.

Proof of Theorem 4.4 Let $f$ be any feedback policy that admits a PBE $\sigma$ for which (14) holds. As in the proof of Theorem 4.2, Jensen's inequality and the law of 
iterated expectation applied to (10) imply that the expected stage 2 effort satisfies

$$
\begin{aligned}
E\left[a_{2}^{*}\left(\sigma_{1}, y\right)\right] & =E\left[\left(c_{2}^{\prime}\right)^{-1}\left(E\left[\phi_{2}\left(x_{1}\right) \mid y\right]\right)\right] \\
& \leq\left(c_{2}^{\prime}\right)^{-1}\left(E\left[E\left[\phi_{2}\left(x_{1}\right) \mid y\right]\right]\right) \\
& =\left(c_{2}^{\prime}\right)^{-1}\left(E\left[\phi_{2}\left(x_{1}\right)\right]\right) \\
& =\left(c_{2}^{\prime}\right)^{-1}\left(\bar{\phi}\left(\sigma_{1}^{1}-\sigma_{1}^{2}\right)\right) \\
& \leq\left(c_{2}^{\prime}\right)^{-1}(\bar{\phi}(0))=a_{2}^{N},
\end{aligned}
$$

where the last inequality follows from Assumption 3. It hence follows from (3) that

$$
v(\sigma, f)=E\left[V\left(\sigma_{1}, a_{2}^{1}=a_{2}^{2}=a_{2}^{*}\left(\sigma_{1}, y\right)\right)\right] \leq V\left(\sigma_{1},\left(a_{2}^{N}, a_{2}^{N}\right)\right) .
$$

Since $\sigma_{1}$ solves (14) by assumption,

$$
V\left(\sigma_{1},\left(a_{2}^{N}, a_{2}^{N}\right)\right) \leq V\left(\left(a_{1}^{*}, a_{1}^{*}\right),\left(a_{2}^{N}, a_{2}^{N}\right)\right)
$$

by Lemma A.2. Since the right-hand side of the above inequality equals the principal's expected payoff in the symmetric PBE under the no-feedback policy, the desired conclusion follows.

Proof of Theorem 4.5 We first show that Assumption 4 implies

$$
\left.\left.P\left(\left|\tilde{\zeta}_{2}\right| \geq \kappa\right)\right)=\min _{\delta \in \mathbf{R}} P\left(\left|\tilde{\zeta}_{2}+\delta\right| \geq \kappa\right)\right) \text { for any } \kappa>0 \text {. }
$$

Let $\delta>0$ and $\kappa>0$ be given. When $\delta<2 \kappa$, we have

$$
\begin{aligned}
& \left.\left.P\left(\left|\tilde{\zeta}_{2}\right|<\kappa\right)\right)-P\left(\left|\tilde{\zeta}_{2}+\delta\right|<\kappa\right)\right) \\
= & -\int_{-\kappa-\delta}^{-\kappa} \phi_{2}(x) d x+\int_{\kappa-\delta}^{\kappa} \phi_{2}(x) d x \\
> & -\delta \phi_{2}(-\kappa)+\delta \phi_{2}(\kappa) \\
= & 0 .
\end{aligned}
$$

On the other hand, when $\delta>2 \kappa$, we have

$$
\begin{aligned}
& \left.\left.P\left(\left|\tilde{\zeta}_{2}\right|<\kappa\right)\right)-P\left(\left|\tilde{\zeta}_{2}+\delta\right|<\kappa\right)\right) \\
= & \int_{-\kappa}^{\kappa} \phi_{2}(x) d x-\int_{-\kappa-\delta}^{\kappa-\delta} \phi_{2}(x) d x \\
> & 2 \kappa \phi_{2}(\kappa)-2 \kappa \phi_{2}(\kappa-\delta) \\
> & 0 .
\end{aligned}
$$


The similar argument proves (23) when $\delta<0$.

We now show that the expected stage 2 effort implied by $\sigma$ is less than or equal to that implied by the symmetric PBE under the full-feedback policy:

$$
E\left[a_{2}^{*}\left(\sigma_{1}, y\right)\right] \leq a_{2}^{F} \equiv \int_{\mathbf{R}^{2}}\left(c_{2}^{\prime}\right)^{-1}\left(\phi_{2}\left(x_{1}\right)\right) \phi_{1}\left(x_{1}\right) d z_{1} .
$$

By the same logic as in the proof of Theorem 4.4, it would then follow from Lemma A.2 that $v(\sigma, f)$ is $\leq$ the principal's expected payoff in the symmetric PBE under the full-feedback policy.

Note that since $E\left[a_{2}^{*}\left(\sigma_{1}, y\right)\right] \leq E\left[\left(c_{2}^{\prime}\right)^{-1}\left(\phi_{2}\left(x_{1}\right)\right)\right]$ as in the proof of Theorem $4.2,(24)$ is implied by

$$
E\left[\left(c_{2}^{\prime}\right)^{-1}\left(\phi_{2}\left(x_{1}\right)\right)\right] \leq a_{2}^{F} .
$$

Let $\eta_{2}:\left[0, \phi_{2}(0)\right] \rightarrow \mathbf{R}_{+}$be the inverse of the restriction of $\phi_{2}$ to $\mathbf{R}_{+}$with $\eta_{2}(0)=\infty$. In other words, for each $u \in\left[0, \phi_{2}(0)\right], \eta_{2}(u) \geq 0$ is the unique number such that $\phi_{2}\left(\eta_{2}(u)\right)=u$. Note that $\eta_{2}$ is well-defined under Assumption 4. Given any $\delta \in \mathbf{R}$, let the function $G(\cdot \mid \delta):\left[0, \phi_{2}(0)\right] \rightarrow \mathbf{R}_{+}$be defined by $G(u \mid \delta)=1-\Phi_{2}\left(\eta_{2}(u)-\delta\right)+$ $\Phi_{2}\left(-\eta_{2}(u)-\delta\right)=P\left(\left|\tilde{\zeta}_{2}+\delta\right| \geq \eta_{2}(u)\right)$. Note that $G(\cdot \mid \delta)$ is a distribution function over $\left[0, \phi_{2}(0)\right]$ since it is increasing, and satisfies $G(0 \mid \delta)=0$ and $G\left(\phi_{2}(0) \mid \delta\right)=1$. If we write $\delta=\sigma_{1}^{1}-\sigma_{1}^{2}$, then

$$
\begin{aligned}
& E\left[\left(c_{2}^{\prime}\right)^{-1}\left(\phi_{2}\left(x_{1}\right)\right) \mid a_{1}\right] \\
= & \int_{\mathbf{R}^{2}}\left(c_{2}^{\prime}\right)^{-1}\left(\phi_{2}\left(x_{1}\right)\right) \phi_{1}\left(x_{1}-\delta\right) d z_{1} \\
= & \int_{0}^{\phi_{2}(0)}\left(c_{2}^{\prime}\right)^{-1}(u) \phi_{1}\left(\eta_{2}(u)-\delta\right)\left(-\eta_{2}^{\prime}(u)\right) d u \\
+ & \int_{0}^{\phi_{2}(0)}\left(c_{2}^{\prime}\right)^{-1}(u) \phi_{1}\left(-\eta_{2}(u)-\delta\right)\left(-\eta_{2}^{\prime}(u)\right) d u \\
= & \int_{0}^{\phi_{2}(0)}\left(c_{2}^{\prime}\right)^{-1}(u) d G(u \mid \delta),
\end{aligned}
$$

where the second equality follows from first dividing the range of the integral and then applying the change of variables from $x_{1}$ to $u=\phi_{2}\left(x_{1}\right)$ or $u=-\phi_{2}\left(x_{1}\right)$. By $(23), G(u \mid \delta)=P\left(\left|\tilde{\zeta}_{2}+\delta\right| \geq \eta_{2}(u)\right) \geq P\left(\left|\tilde{\zeta}_{2}\right| \geq \eta_{2}(u)\right)=G(u \mid 0)$ for any $u \in\left[0, \phi_{2}(0)\right]$ and $\delta \in \mathbf{R}$ so that $G(u \mid 0)$ first-order stochastically dominates $G(u \mid \delta)$ with $\delta \neq 0$. Since $\left(c_{2}^{\prime}\right)^{-1}$ is increasing, it follows that

$$
\int_{0}^{\phi_{2}(0)}\left(c_{2}^{\prime}\right)^{-1}(u) d G(u \mid \delta) \leq \int_{0}^{\phi_{2}(0)}\left(c_{2}^{\prime}\right)^{-1}(u) d G(u \mid 0) .
$$


Changing variables back to $x_{1}$, we see that the right-hand side of this inequality equals $a_{2}^{F}$.

\section{A.2 Non-differential Feedback Policies}

The analysis of optimal policies in Section 4 partially extends to a class of feedback policies that are not necessarily a function of the difference $x_{1}$ alone. For this, we suppose that $f: \mathbf{R}^{2} \rightarrow Y$ is a function of the performance profile $z_{1}=\left(z_{1}^{1}, z_{1}^{2}\right)$ and for any $y \in Y$, let $f^{-1}(y)=\left\{z_{1} \in \mathbf{R}^{2}: f\left(z_{1}\right)=y\right\}$ denote the inverse image of $\{y\}$. For any set $A \subset \mathbf{R}^{2}$ and $b \in \mathbf{R}^{2}$, denote $A+b=\{a+b: a \in A\}$ and $-A=\{-a: a \in A\}$. The alternative conditions on $f$ are as follows:

Assumption 5. Let $z_{1}, \hat{z}_{1} \in \mathbf{R}^{2}$ and $s \in \mathbf{R}$.

$$
\begin{aligned}
& \text { 1. } f\left(z_{1}\right)=f\left(\hat{z}_{1}\right) \Rightarrow f\left(z_{1}-(s, s)\right)=f\left(\hat{z}_{1}-(s, s)\right) \text {. } \\
& \text { 2. } f^{-1}\left(f\left(z_{1}\right)\right)=-f^{-1}\left(f\left(-z_{1}\right)\right) \text {. }
\end{aligned}
$$

Intuitively, the first condition states that each level curve of $f$ can be obtained by shifting another level curve in the direction of the 45-degree line. The second condition is the generalization of symmetry defined in the text. Any symmetric feedback policy $f$ that is a function of $x_{1}$ alone satisfies Assumption 5. Other examples of feedback policies that satisfy Assumption 5 are:

$$
f\left(z_{1}\right)=\frac{z_{1}^{1}+z_{1}^{2}}{2} \text { and } \quad f\left(z_{1}\right)=z_{1}^{1} .
$$

Assumption 6. The joint density $\xi_{1}$ of $\varepsilon_{1}$ satisfies $\xi_{1}\left(\varepsilon_{1}\right)=\xi_{1}\left(-\varepsilon_{1}\right)$ for any $\varepsilon_{1} \in$ $\mathbf{R}^{2}$.

Assumption 6 holds when, say, $\varepsilon_{1}$ has the normal distribution $N(0, \Sigma)$ with $\Sigma=\sigma^{2}\left(\begin{array}{ll}1 & \rho \\ \rho & 1\end{array}\right)$. When $f$ is a function of $x_{1}$ alone, the key observations are that the first-order conditions for the stage 1 effort in (14) can be rewritten as in (7), and that the strategic effect equals zero for symmetric policies. The next lemma shows that the same holds under Assumptions 5 and 6.

Lemma A.3. Suppose that Assumptions 1, 5 and 6 hold. There exists a unique symmetric PBE $\sigma$. Furthermore, $\sigma_{1}^{i}=a_{1}^{*}$ and $\sigma_{2}^{i}(y)=a_{2}^{*}\left(\sigma_{1}, y\right)$ for $y \in Y$ and $i=1,2$. 
Proof. Let $\sigma_{1} \in \mathbf{R}_{+}^{2}$ be such that $\sigma_{1}^{1}=\sigma_{1}^{2}$. With the change of variables $\tilde{z}_{1}=z_{1}-\sigma_{1}$, the integral in the first equation of (14) becomes

$$
\int_{\mathbf{R}^{2}} c_{2}\left(a_{2}^{*}\left(\sigma_{1}, f\left(\tilde{z}_{1}+\sigma_{1}\right)\right)\right) \frac{\partial \xi_{1}}{\partial \varepsilon_{1}^{1}}\left(\tilde{z}_{1}\right) d \tilde{z}_{1}
$$

Hence, we have

$$
\begin{aligned}
c_{2}^{\prime}\left(a_{2}^{*}\left(\sigma_{1}, f\left(\tilde{z}_{1}+\sigma_{1}\right)\right)\right) & =E_{z_{1}}\left[\phi_{2}\left(x_{1}\right) \mid z_{1} \in f^{-1}\left(f\left(\tilde{z}_{1}+\sigma_{1}\right)\right)\right] \\
& =E_{\varepsilon_{1}}\left[\phi_{2}\left(\varepsilon_{1}^{1}-\varepsilon_{1}^{2}\right) \mid \varepsilon_{1} \in f^{-1}\left(f\left(\tilde{z}_{1}+\sigma_{1}\right)\right)-\sigma_{1}\right] \\
& =E_{\varepsilon_{1}}\left[\phi_{2}\left(\varepsilon_{1}^{1}-\varepsilon_{1}^{2}\right) \mid \varepsilon_{1} \in f^{-1}\left(f\left(\tilde{z}_{1}\right)\right)\right] \\
& =\frac{\int_{f^{-1}\left(f\left(\tilde{z}_{1}\right)\right)} \phi_{2}\left(\varepsilon_{1}^{1}-\varepsilon_{1}^{2}\right) \phi_{1}\left(\varepsilon_{1}\right) d \varepsilon_{1}}{\int_{f^{-1}\left(f\left(\tilde{z}_{1}\right)\right)} \phi_{1}\left(\varepsilon_{1}\right) d \varepsilon_{1}},
\end{aligned}
$$

where the second equality uses $z_{1}=\sigma_{1}+\varepsilon_{1}$ and $\sigma_{1}^{1}=\sigma_{1}^{2}$, and the third equality follows from the first condition of Assumption 5 since it is equivalent to ${ }^{25}$

$$
f^{-1}\left(f\left(z_{1}+(s, s)\right)\right)=f^{-1}\left(f\left(z_{1}\right)\right)+(s, s) \text { for any } z_{1} \in \mathbf{R}^{2} \text { and } s \in \mathbf{R} \text {. }
$$

Since the last quantity is independent of $\sigma_{1}$, so is $c_{2}\left(a_{2}^{*}\left(\sigma_{1}, f\left(\tilde{z}_{1}+\sigma_{1}\right)\right)\right)$, which we denote by $m\left(\tilde{z}_{1}\right)$. By Assumptions 5 and 6 , we have for any $z_{1} \in \mathbf{R}^{2}$,

$$
m\left(z_{1}\right)=m\left(-z_{1}\right)
$$

and

$$
\frac{\partial \xi_{1}}{\partial \varepsilon_{1}^{1}}\left(z_{1}\right)=-\frac{\partial \xi_{1}}{\partial \varepsilon_{1}^{1}}\left(-z_{1}\right)
$$

Now express (26) as

$$
\begin{aligned}
& \int_{\mathbf{R}^{2}} m\left(z_{1}\right) \frac{\partial \xi_{1}}{\partial \varepsilon_{1}^{1}}\left(z_{1}\right) d z_{1} \\
& =\int_{z_{1}^{1}>0, z_{1}^{2}>0} m\left(z_{1}\right) \frac{\partial \xi_{1}}{\partial \varepsilon_{1}^{1}}\left(z_{1}\right) d z_{1}+\int_{z_{1}^{1}>0, z_{1}^{2}<0} m\left(z_{1}\right) \frac{\partial \xi_{1}}{\partial \varepsilon_{1}^{1}}\left(z_{1}\right) d z_{1} \\
& +\int_{z_{1}^{1}<0, z_{1}^{2}>0} m\left(z_{1}\right) \frac{\partial \xi_{1}}{\partial \varepsilon_{1}^{1}}\left(z_{1}\right) d z_{1}+\int_{z_{1}^{1}<0, z_{1}^{2}<0} m\left(z_{1}\right) \frac{\partial \xi_{1}}{\partial \varepsilon_{1}^{1}}\left(z_{1}\right) d z_{1} .
\end{aligned}
$$

${ }^{25}(\Rightarrow)$ Suppose $\tilde{z}_{1} \in f^{-1}\left(f\left(z_{1}+(s, s)\right)\right.$. Then $f\left(\tilde{z}_{1}\right)=f\left(z_{1}+(s, s)\right)$ and hence $f\left(\tilde{z}_{1}-(s, s)\right)=f\left(z_{1}\right)$ by Assumption 5(1). This implies $\tilde{z}_{1}-(s, s) \in f^{-1}\left(f\left(z_{1}\right)\right)$ or equivalently, $\tilde{z}_{1} \in f^{-1}\left(f\left(z_{1}\right)\right)+(s, s)$. On the other hand, if $\tilde{z}_{1} \in f^{-1}\left(f\left(z_{1}\right)\right)+(s, s)$, then $f\left(\tilde{z}_{1}-(s, s)\right)=f\left(z_{1}\right)$ so that $f\left(\tilde{z}_{1}\right)=f\left(z_{1}+\right.$ $(s, s))$. This implies that $\tilde{z}_{1} \in f^{-1}\left(f\left(z_{1}+(s, s)\right)\right)$. $(\Leftarrow)$ Suppose $f\left(z_{1}\right)=f\left(\hat{z}_{1}\right)$ but $f\left(z_{1}-(s, s)\right) \neq$ $f\left(\hat{z}_{1}-(s, s)\right)$ for some $z_{1}, \hat{z}_{1}$, and $s$. Write $\tilde{z}_{1}=\hat{z}_{1}-(s, s)$. Then $z_{1} \in f^{-1}(f(\tilde{z}+(s, s)))$. However, $z_{1}-(s, s) \notin f^{-1}\left(\tilde{z}_{1}\right)$ so that $z_{1} \notin f^{-1}\left(f\left(\tilde{z}_{1}\right)\right)+(s, s)$. 
Applying the change of variables $\hat{z}_{1}=-z_{1}$ to the third and fourth integrals and then using (27) and (28), we obtain

$$
\begin{aligned}
& \int_{\mathbf{R}^{2}} m\left(z_{1}\right) \frac{\partial \xi_{1}}{\partial \varepsilon_{1}^{1}}\left(z_{1}\right) d z_{1} \\
& =\int_{z_{1}^{1}>0, z_{1}^{2}>0} m\left(z_{1}\right) \frac{\partial \xi_{1}}{\partial \varepsilon_{1}^{1}}\left(z_{1}\right) d z_{1}+\int_{z_{1}^{1}>0, z_{1}^{2}<0} m\left(z_{1}\right) \frac{\partial \xi_{1}}{\partial \varepsilon_{1}^{1}}\left(z_{1}\right) d z_{1} \\
& +\int_{\hat{z}_{1}^{1}>0, \hat{z}_{1}^{2}<0} m\left(-\hat{z}_{1}\right) \frac{\partial \xi_{1}}{\partial \varepsilon_{1}^{1}}\left(-\hat{z}_{1}\right) d \hat{z}_{1}+\int_{\hat{z}_{1}^{1}>0, \hat{z}_{1}^{2}>0} m\left(-\hat{z}_{1}\right) \frac{\partial \xi_{1}}{\partial \varepsilon_{1}^{1}}\left(-\hat{z}_{1}\right) d \hat{z}_{1} \\
& =0 .
\end{aligned}
$$

The symmetric argument proves that the integral in the second equation of (14) also equals zero. This shows that (14) has a unique symmetric solution $\sigma_{1}^{1}=\sigma_{1}^{2}=\bar{\phi}(0)$. Hence, by Theorem 3.2, there exists a unique symmetric PBE $\sigma$ under $f$ and $\sigma$ is as specified in the lemma.

Corollary A.4. Suppose that Assumptions 1 and 6 hold. Among the class of feedback policies that satisfy Assumption 5, the no-feedback policy maximizes $\bar{v}^{*}$ if $c_{2}^{\prime}$ is convex and the full-feedback policy maximizes $\bar{v}^{*}$ if $c_{2}^{\prime}$ is concave.

\section{References}

[1] Abreu, D., Milgrom, P., Pearce, D., 1991, Information and timing in repeated partnerships, Econometrica, 59, 1713-1733.

[2] Aoyagi, M., 2006, Information feedback in a dynamic tournament, working paper; ISER, Osaka University, http://www.eonet.ne.jp/ aoyagi/ feedback2006.pdf.

[3] de-Frutos, M.-A., Rosenthal,R., 1998, On some myths about sequenced common-value auctions, Games Econ. Behav. 23, 201-21.

[4] Ederer, F., 2004, Feedback and motivation in dynamic tournaments, working paper; MIT.

[5] Fudenberg, D., Gilbert, R., Stiglitz, J., Tirole, J., 1983, Preemption, leapfrogging, and competition in patent races, Europ. Econ. Rev. 22, 3-31.

[6] Fudenberg, D., Tirole, J., 1991, Game Theory, MIT Press: Cambridge, MA. 
[7] Gershkov, A., Perry, M., 2006, Tournaments with midterm reviews, working paper; University of Bonn and Hebrew University.

[8] Glazer, D., Hassin, R., 1988, Optimal contests, Econ. Inquiry 26, 133-43.

[9] Goltsman, M., Mukherjee, A., 2006, Information disclosure in multistage tournaments, working paper; University of Western Ontario and Bates White LLC.

[10] Gradstein, M., Konrad, K. A., 1999, Orchestrating rent seeking contests, Econ. J. 109, 536-45.

[11] Green, J., Stokey, N., 1983, A comparison of tournaments and contracts, J. Polit. Economy 91, 349-364.

[12] Harris, C., Vickers, C., 1985, Perfect equilibrium in a model of a race, Rev. Econ. Stud. 52, 193-209.

[13] Kaplan, T., Zamir, S., 2000, The strategic use of seller information in privatevalue auctions, working paper; University of Exeter and the Hebrew University of Jerusalem.

[14] Kimball, M., 1990, Precautionary saving in the small and in the large, Econometrica 58, 53-73.

[15] Lazear, E., Rosen, S., 1981, Rank order tournaments as optimal labor contracts, J. Polit. Economy 89, 841-64.

[16] Lizzeri, A., Meyer, M., Persico, N., 2002, The incentive effects of interim performance evaluations, CARESS Working Paper 02-09.

[17] Milgrom, P. R., Weber, R., 1982, A theory of auctions and competitive bidding, Econometrica 50, 1059-1122.

[18] Moldovanu, B., Sela, A., 2001, The optimal allocation of prizes in contests, Amer. Econ. Rev. 91, 542-58.

[19] Moldovanu, B., Sela, A., 2006, Contests Architecture, J. Econ. Theory 126, 70-96.

[20] Nalebuff, B. J., Stiglitz, J. E., 1983, Prizes and incentives: towards a general theory of compensation and competition, Bell J. Econ. 14, 21-43. 
[21] Perry, M., Reny, P. J., 1999, On the failure of the linkage principle in multi-unit auctions, Econometrica 67, 895-900.

[22] Radner, R., 1985, Repeated principal-agent games with discounting, Econometrica 53, 1173-98.

[23] Rogerson, W., 1985, Repeated moral hazard, Econometrica 53, 69-76.

[24] Williams, R., 1998, Performance Management: Perspectives on Employee Performance, International Thomson Business Press, London.

[25] Yildirim, H., 2005, Contests with multiple rounds, Games Econ. Behav. 51, 213-227.

[26] Wang, R., Zhang, J., 2007, The role of information revelation in elimination contests, working paper; Queens University. 